\title{
Periconceptional alcohol exposure causes female-specific perturbations to trophoblast differentiation and placental formation in the rat
}

\author{
Jacinta I. Kalisch-Smith ${ }^{1}$, Sarah E. Steane ${ }^{1}$, David G. Simmons ${ }^{1}$, Marie Pantaleon ${ }^{1}$, Stephen T. Anderson ${ }^{1}$, \\ Lisa K. Akison ${ }^{1,2}$, Mary E. Wlodek ${ }^{3}$ and Karen M. Moritz ${ }^{1,2, *}$
}

\begin{abstract}
The development of pathologies during pregnancy, including preeclampsia, hypertension and fetal growth restriction (FGR), often originates from poor functioning of the placenta. In vivo models of maternal stressors, such as nutrient deficiency, and placental insufficiency often focus on inadequate growth of the fetus and placenta in late gestation. These studies rarely investigate the origins of poor placental formation in early gestation, including those affecting the pre-implantation embryo and/or the uterine environment. The current study characterises the impact on blastocyst, uterine and placental outcomes in a rat model of periconceptional alcohol exposure, in which $12.5 \%$ ethanol is administered in a liquid diet from 4 days before until 4 days after conception. We show female-specific effects on trophoblast differentiation, embryo-uterine communication, and formation of the placental vasculature, resulting in markedly reduced placental volume at embryonic day 15 . Both sexes exhibited reduced trophectoderm pluripotency and global hypermethylation, suggestive of inappropriate epigenetic reprogramming. Furthermore, evidence of reduced placental nutrient exchange and reduced pre-implantation maternal plasma choline levels offers significant mechanistic insight into the origins of FGR in this model.
\end{abstract}

KEY WORDS: Placenta, Development, Trophoblast, Alcohol, Pre-implantation

\section{INTRODUCTION}

Formation of the placenta is vital to healthy development of the fetus; perturbation of placental formation can have long-lasting effects into adult life, through greater susceptibility to adulthood disease (Burton et al., 2016). Poor functioning of the placenta, particularly by placental malperfusion, is involved in the development of pregnancyassociated conditions such as hypertension, pre-eclampsia, fetal growth restriction (FGR) and miscarriage (Burton et al., 2016). The origins of uteroplacental insufficiency and these associated pathologies have long been suspected to begin at implantation (Kajantie et al., 2010; Roberts and Cooper, 2001; Fisher, 2004;

\footnotetext{
${ }^{1}$ School of Biomedical Sciences, Faculty of Medicine, The University of Queensland, St Lucia, QLD 4072, Australia. ${ }^{2}$ Child Health Research Centre, The University of Queensland, South Brisbane, QLD 4101, Australia. ${ }^{3}$ Department of Physiology, School of Biomedical Sciences, Faculty of Medicine, Dentistry and Health Sciences, The University of Melbourne, Parkville, Victoria 3010, Australia.

*Author for correspondence (k.moritz1@uq.edu.au)
}

(D) J.I.K.-S., 0000-0002-5071-3805; S.E.S., 0000-0001-8594-3486; D.G.S., 00000002-4115-9371; M.P., 0000-0002-5659-2354; L.K.A., 0000-0002-2667-3743; M.E.W., 0000-0002-8490-9099; K.M.M., 0000-0002-8085-0034

Received 23 September 2018; Accepted 18 April 2019
Burton and Jauniaux, 2010). However, evidence is currently lacking, particularly in animal models, to explain their aetiology from early in gestation.

The most common of these pathologies, FGR, is the result of poor placentation in response to a perturbed maternal environment, with the severity of the outcome dependent on both the time of exposure to stress and the feto-placental sex (reviewed by KalischSmith et al., 2017a). Central to this study, is maternal alcohol consumption. Chronic alcohol exposure during human gestation is associated with low birth weight (Jones and Smith, 1973; Manning and Eugene Hoyme, 2007; O'Leary et al., 2009; Tai et al., 2016), as well as placental complications, including miscarriage, preeclampsia, pre-term birth and stillbirth (Kaminski et al., 1978; Tai et al., 2016; Maconochie et al., 2007; Avalos et al., 2014; Salihu et al., 2011; Meyer-Leu et al., 2011; Marbury et al., 1983). However, one period that is potentially susceptible to alcohol exposure and has received little attention, is consumption prior to pregnancy recognition. Approximately $47 \%$ of women drink around conception, with $15-39 \%$ of these reported as receiving high doses of more than five standard drinks on one occasion (Colvin et al., 2007; Wallace et al., 2007; Muggli et al., 2016). Interestingly, 87\% of women cease drinking after pregnancy recognition (Muggli et al., 2016). Given that $50 \%$ of pregnancies are unplanned (Colvin et al., 2007; Finer and Zolna, 2016), the periconceptional window, which includes early embryogenesis, may be more highly exposed to alcohol than is currently appreciated.

This critical periconceptional period includes important events prior to placentation, including oocyte maturation, fertilisation, embryonic genome activation, as well as epigenetic and metabolic changes prior to formation of the blastocyst (reviewed by Fleming et al., 2004). The blastocyst contains the stem cell populations for both the embryo [the inner cell mass (ICM)] and the placenta [the trophectoderm (TE)]. The TE gives rise to unique trophoblast subtypes that facilitate invasion into the uterus and establish the definitive chorioallantoic placenta for nutrient exchange (Watson and Cross, 2005). Indeed, periconceptional insults, such as undernutrition (Kwong et al., 2000, 2006) and in vitro fertilisation (Bloise et al., 2012; Tan et al., 2016a,b), have been shown to impact these early stem cell populations and result in subsequent changes to placentation and fetal growth. In addition, male and female pre-implantation embryos formed in vivo have the same capacity to form blastocysts and have equal potential to implant, with differences in placental development only being apparent from mid-gestation (Kalisch-Smith et al., 2017b). However, it is not known whether maternal perturbations impact male and female embryos in the same way, and if they lead progressively to sex-specific alterations and adaptations in later development. 
We have recently characterised a model of periconceptional alcohol (PC-EtOH) exposure in the rat, which exhibits FGR, sex-specific alterations in the late-gestation placenta and compromised metabolic outcomes in adulthood (Gårdebjer et al., 2014, 2015). We examined the cellular origin of FGR, and whether this derives from a direct effect of alcohol on pre-implantation development and/or the maternal environment, or is due to poor placental formation. Both we (Kalisch-Smith et al., 2016) and others (Bolnick et al., 2018) have previously shown direct effects on trophoblast proliferation and differentiation in culture, but have not considered other influences mediated by development in vivo. Here, we report embryonic and placental outcomes across gestation after PC-EtOH exposure in vivo, specifically on male and female preimplantation embryos, and demonstrate comprehensive stage-specific developmental defects that could affect embryonic competence and fetal growth by late gestation. We also highlight perturbation of both waves of trophoblast invasion, a previously undescribed phenotype in other models of nutritional programming.

\section{RESULTS}

PC-EtOH causes sex-specific alterations to pre-implantation development and trophoblast differentiation

To evaluate the effect of PC-EtOH on blastocyst formation, uteri from rat dams were flushed on the morning of embryonic day (E) 5 and assessed for the number of embryos at specific developmental stages. Relative to control, $\mathrm{PC}-\mathrm{EtOH}$ exposure had no significant effect on the number of embryos reaching the blastocyst stage (Table S1). We next investigated cell counts in E5 embryos and used immunofluorescent labelling of CDX2 to differentiate TE (CDX2 positive) from ICM (CDX2 negative). When all individual embryos (unsexed) were assessed, no differences were found between treatments for any embryo parameter: total cell count, TE or ICM count, TE:ICM or \% ICM (Fig. S1). No differences were found in total blastocyst cell count or TE counts after PC-EtOH (Fig. 1A,B). Similarly, no changes to ICM cell count or the ratio of TE to ICM cells were shown after PC-EtOH (Fig. 1C,D). When blastocysts were quantified for nuclear CDX2 fluorescence in TE cells, a measure of TE pluripotency (Strumpf et al., 2005), PC-EtOH markedly reduced levels in both males and females $(P \operatorname{Trt}<0.001$; Fig. 1F, Fig. S2). Considering the marked changes in the epigenome found after alcohol exposure in other studies (reviewed by KalischSmith and Moritz, 2017), global levels of 5-methylcytosine (5mC) and 5-hydroxymethylcytosine $(5 \mathrm{hmC})$ were assessed by semiquantitative immunofluorescence. Both male and female PC-EtOH embryos showed marked increases in nuclear staining for $5 \mathrm{mC}$ in TE $(P \operatorname{Trt}<0.01$; Fig. $1 \mathrm{G}, \mathrm{K}, \mathrm{L}$, Fig. S3) and ICM nuclei $(P \operatorname{Tr} \mathrm{C}<0.01$; Fig. $1 \mathrm{H})$, suggesting that alcohol causes alterations in DNA methylation and appropriate epigenetic reprogramming may be reduced or delayed. No differences were found between treatments or sex for 5hmC (Fig. 1I,J, Fig. S4).

Given the dramatic change in CDX2 and $5 \mathrm{mC}$ levels, the TE was investigated for its ability to differentiate and grow in culture after in vivo $\mathrm{PC}-\mathrm{EtOH}$. Following 6 days of culture, embryos exposed to $\mathrm{PC}-\mathrm{EtOH}$ had reduced outgrowth area and number of trophoblasts in both sexes $(P<0.05$; Fig. 2A,B). However, when assessed for the presence of the largest trophoblast subtype; equivalent of the parietal trophoblast giant cells (P-TGCs) in vivo, $\mathrm{PC}-\mathrm{EtOH}$ females showed a subtle reduction in the number of these cells when
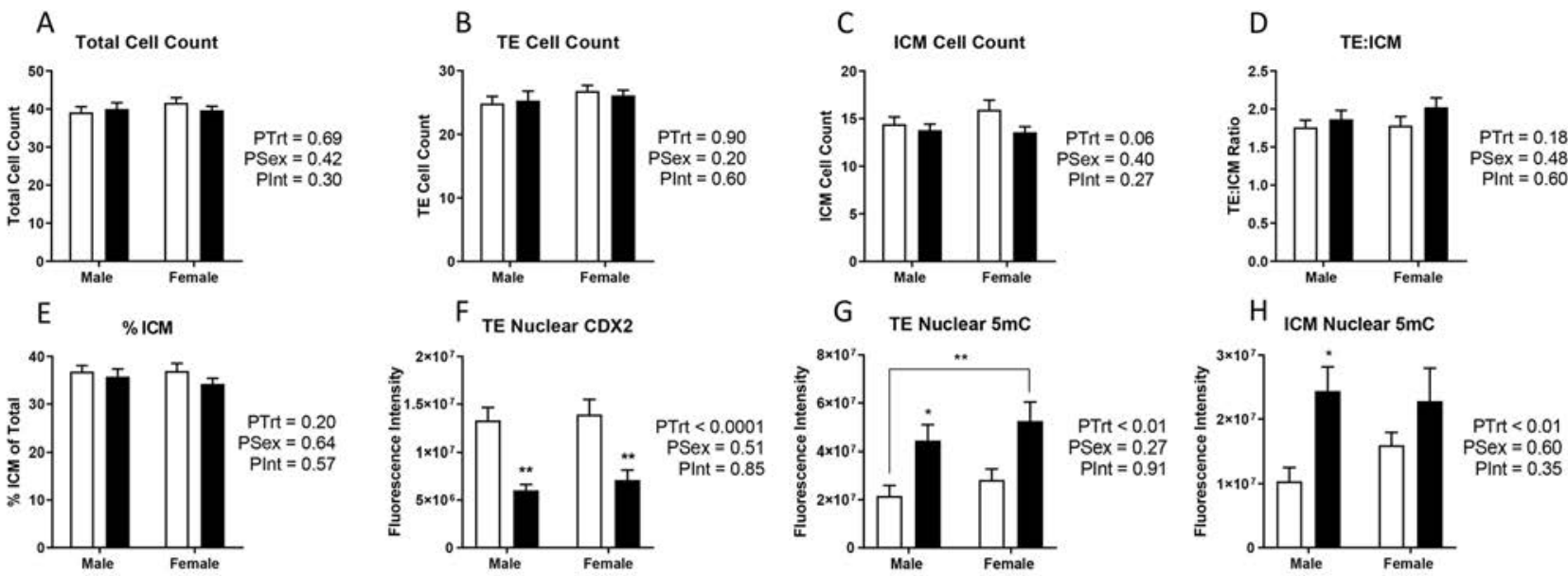

G TE Nuclear $5 \mathrm{mC}$
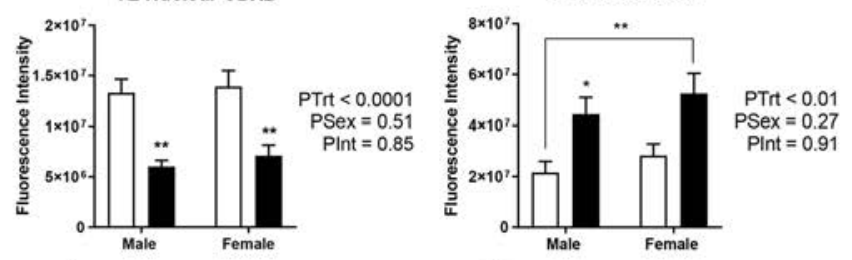

H ICM Nuclear $5 \mathrm{mC}$

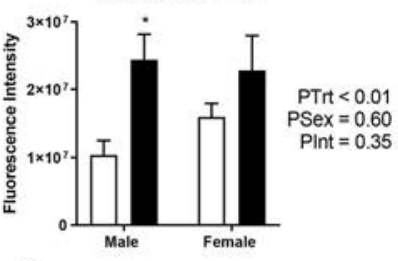

I TE Nuclear $5 \mathrm{hmc}$
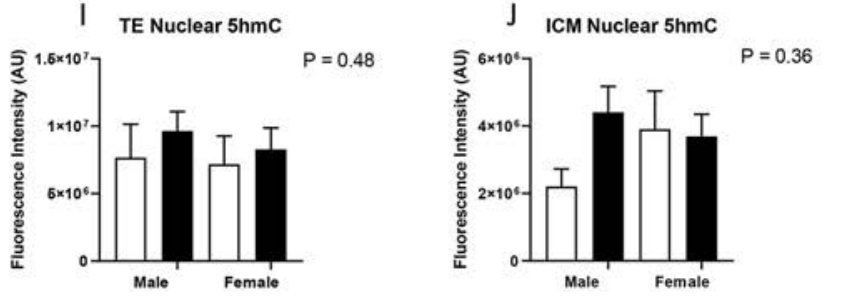

K

$\mathrm{L}$
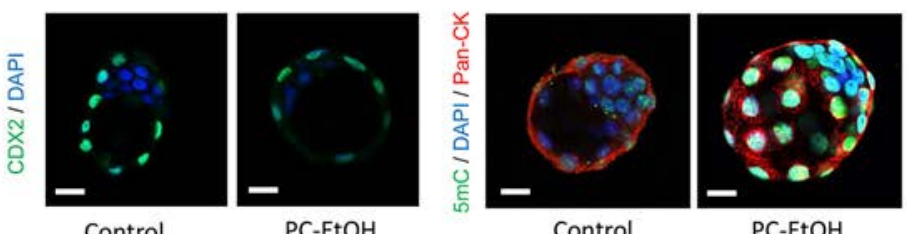

Fig. 1. Effects of in vivo PC-EtOH exposure on cell number, allocation and differentiation potential in E5 rat blastocysts. (A-E) Total blastocyst cell count (A), TE cell count (B), ICM cell count (C), ratio of TE to ICM (D) and percentage ICM count of total cell count (E) were unchanged in E5 rat blastocysts after PC-EtOH exposure. (F) Nuclear CDX2 fluorescence intensity was significantly reduced in both male and female blastocysts after PC-EtOH exposure. (G-L) Nuclear expression of $5 \mathrm{mC}(\mathrm{G}, \mathrm{H})$ and $5 \mathrm{hmC}(\mathrm{I}, \mathrm{J})$ was increased in $\mathrm{PC}-\mathrm{EtOH}$ males and females in TE and ICM cells. (K,L) Representative images of control and PC-EtOH blastocysts for CDX2 (K) and $5 \mathrm{mC}(\mathrm{L})$. Blastocysts localised with $5 \mathrm{mC}$ were counterstained with Pan-CK to localise TE cells. (A-H) Two-way ANOVA with Tukey's post-hoc tests. (I,J) Non-parametric Kruskal-Wallis tests. Embryos were pooled from each litter for analyses. Control (white bars) and PC-EtOH exposed (black bars) are is shown. (A-E) $n=14-26$ embryos/sex from 5-7 litters. (F-J) $n=5-12$ embryos/sex from 2 litters/treatment. Assays for each marker were performed separately. All data are presented as mean \pm s.e.m. Significance was set at $P<0.05$. ${ }^{*} P<0.05,{ }^{* *} P<0.01, P$ Int, $P$ interaction. Scale bars: $20 \mu$ m. 

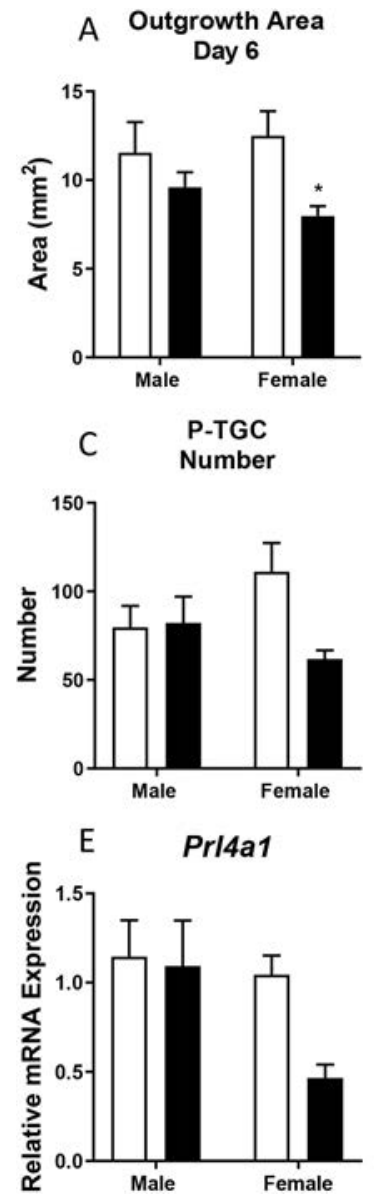

$\mathrm{PTrt}=0.13$

PSex $=0.72$

$\mathrm{PInt}=0.09$

$\begin{aligned} \text { PTrt } & <0.05 \\ \text { PSex } & =0.79\end{aligned}$

PInt $=0.31$
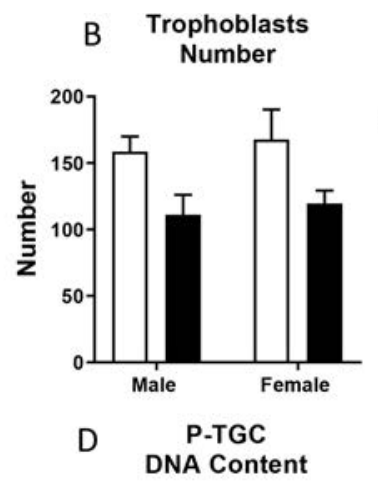

PTrt $<0.05$

PSex $=0.62$

PInt $=0.98$

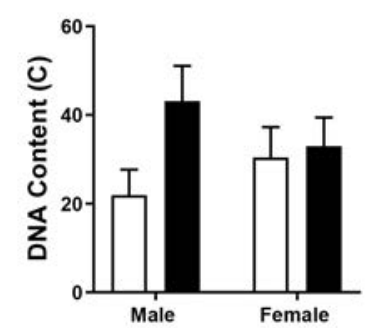

Fig. 2. In vivo PC-EtOH exposure reduces trophoblast outgrowth capacity. (A-E) E5 in vivo-exposed embryos were cultured to day 6 and assessed for outgrowth area $(\mathrm{A})$, trophoblast number $\left(>200 \mu \mathrm{m}^{2}\right)(\mathrm{B})$, number of P-TGCs $\left(>1000 \mu \mathrm{m}^{2}\right)(\mathrm{C})$ and DNA content of P-TGCs (D). Gene expression profile for Prl4a1 (communication with dNK cells, TGC specific expression) in outgrowths were also assessed (E). Control (white bars) and $\mathrm{PC}-\mathrm{EtOH}$ exposed (black bars) data are shown. All data are presented as mean \pm s.e.m. and analysed by two-way ANOVA. (F,G) Representative images of immunofluorescent localisation of trophoblasts with pan-cytokeratin (red), and DAPI (blue) for control female (F) and PC-EtOH female (G) outgrowths. Gene expression is plotted relative to the control male group, and standardised to the geometric mean of two housekeepers (18S and Rp/13a). Gene expression assays were carried out in duplicate. (A) $n=15-25$ from 4 litters/treatment. (B-D) $n=6-11$ per sex from 4 litters/treatment. (E) $n=7-10$ from 3-4 litters/treatment. Scale bars: $100 \mu \mathrm{m}$. analysed separately from males (Fig. 2C, Fig. S5G; see Fig. 2F, G for representative images of these outgrowths). P-TGC DNA content, a marker of ploidy, showed no differences between treatments or sex (Fig. 2D). Gene expression analysis showed no alteration in the expression levels of the trophoblast stem cell markers Elf5 or Eomes, the P-TGC marker Prl3d1, the downstream ectoplacental cone and chorion genes Ascl2 and Hand1, or the X chromosome inactivation gene Xist, following PC-EtOH (Fig. S5A-F). Analysis of $\mathrm{PC}$-EtOH-exposed outgrowths showed a reduction in expression of Prl4al in females analysed separately from males (Fig. 2E, Fig. $5 \mathrm{H} ; P<0.01)$. Prl4al is a highly endoreplicated gene expressed exclusively by TGCs for communication with maternal decidual natural killer (dNK) cells (Ain et al., 2004; Müller et al., 1999; Hannibal and Baker, 2016).

\section{PC-EtOH causes subtle changes to uterine decidualisation}

Following embryonic characterisation, $\mathrm{PC}-\mathrm{EtOH}$ was investigated for potential influences on the uterine environment and the establishment and maintenance of pregnancy. Firstly, maternal oestrogen and progesterone concentrations were quantified over the peri-implantation period (E5-E7) and no notable changes were observed after PC-EtOH (Fig. 3A,B). E5 uteri and E7 early postimplantation sites were then assessed for expression of genes involved in the hormone-dependent processes of receptivity and decidualisation. PC-EtOH caused a change in gene expression of the oestrogen receptor $(E s r l)(P \operatorname{Tr}<<0.05)$ that was time dependent $(P$ Int $<0.01$; Fig. 3C). Post-hoc analysis demonstrated that $E s r l$ was reduced at E5 following $\mathrm{PC}-\mathrm{EtOH}(P<0.01)$, but unaltered at E7. Western immunoblotting for oestrogen receptor A (ESR1) showed a
$33 \%$ reduction in protein expression, although this did not reach statistical significance $(P=0.08$; Fig. 3G). No change to the expression of receptor Esr2 (Table S2) was observed. Similarly, no changes to downstream oestrogen response genes (Hbegf, Vegfa, Lif, Fgf2, Fgf9; Table S2) or receptivity-associated genes [Lif, Ihh or Usagl (Sostdc1)] were found. However, progesterone receptor $(P g r)$ expression was time and treatment dependent, with post-hoc analysis showing expression was reduced at E5 $(P<0.01)$ and increased at E7 after PC-EtOH $(P<0.01$; Fig. 3D). Consistent with this, the progesterone-associated decidualisation genes Bmp2 and Coup-TFII (Nr2f2) were decreased by PC-EtOH at E5 only (Fig. 3E,F), with no other changes found for Ihh, Areg, Hand2 or Igfbpl (Table S2). Further histological characterisation of E7 implantation sites showed no gross changes to stromal or decidual volume (Fig. 3J,K, see 3I,J for representative images). Expression of Prf1, a dNK cell marker, remained unchanged (Table S2).

\section{PC-EtOH reduces dNK cell infiltration and trophoblast invasion in placentae of female embryos}

In situ hybridization for Prfl in placentae at E11 showed that PC-EtOH-exposed placentae from females had increased PrfI expression localised to dNK cells in the decidual region, suggestive of delayed dNK cell homing and activation (Fig. 4A). The dNK cells are important in mediating the second wave of trophoblast invasion, primarily the spiral artery trophoblast giant cells (SpA-TGCs). These cells remodel the maternal spiral arteries into low-resistance, highflow vessels, allowing increased labyrinthine blood supply (Chakraborty et al., 2011; Wallace et al., 2012). Thus, we proceeded to investigate invasion at E13, E15 and E20 after 

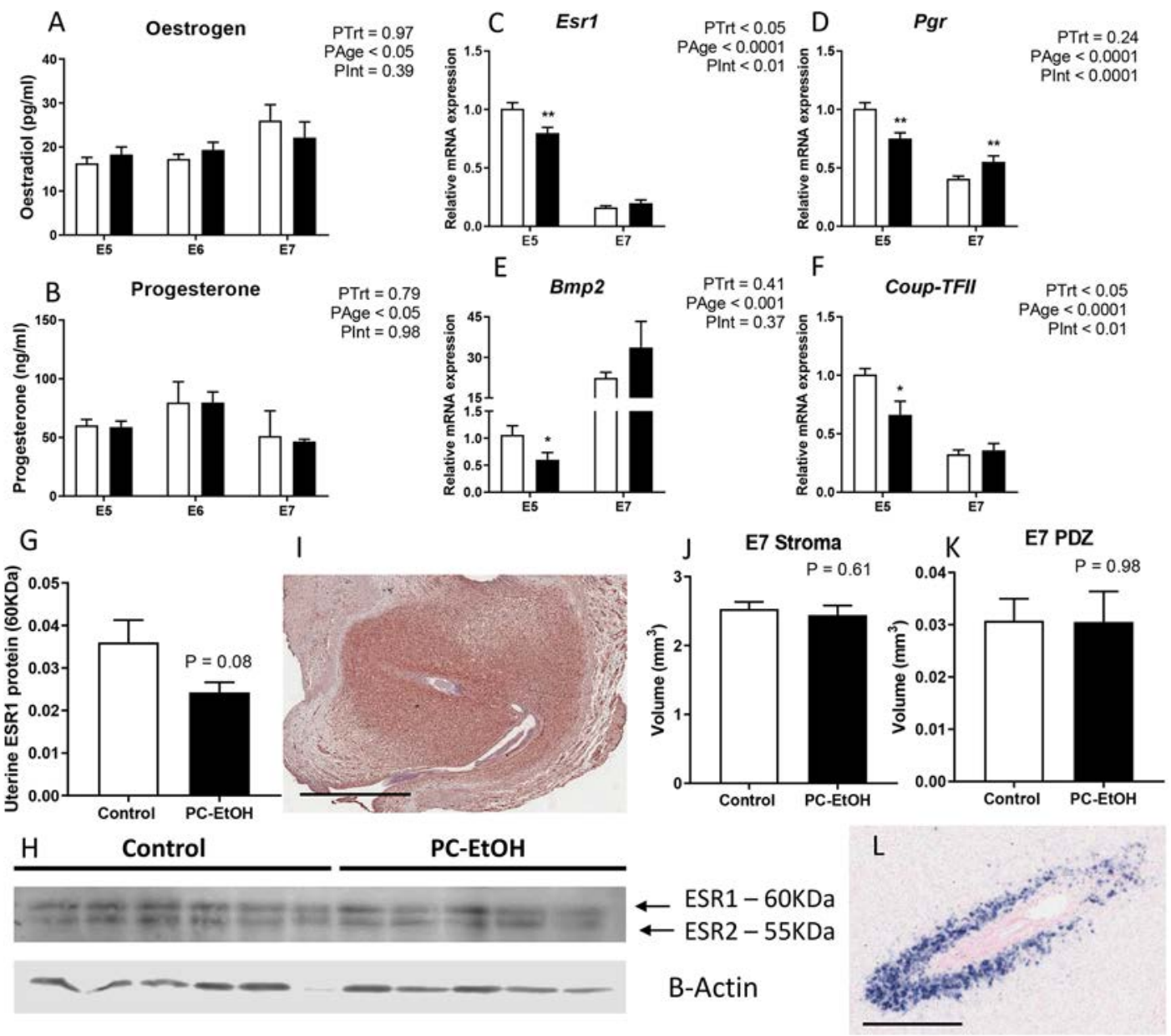

Fig. 3. PC-EtOH exposure affects sex-hormone receptor expression but does not affect long term decidualisation. (A,B) Oestrogen (A) and progesterone (B) levels in maternal plasma were assessed by radioimmunoassay over the peri-implantation period (E5-E7). (C-F) Gene expression assays on uterine samples during receptivity (E5) and post-implantation (E7) decidualisation, to assess expression of oestrogen receptor alpha (Esr1) (C), progesterone receptor $(P g r)(D)$, and the progesterone responsive decidualisation genes Bmp2 $(\mathrm{E})$ and Coup-TFII $(\mathrm{F})$. $(\mathrm{G}, \mathrm{H})$ Representative western blot $(\mathrm{H})$ and densitometric analysis (G) for ESR1 on E5 uteri, normalised to $\beta$-actin. (I) Immunolocalization of vimentin at the implantation site at E7 to identify uterine stromal cells. (J) Stromal volume evaluated at E7. (K) Primary decidual volume (PDZ) at E7. (L) In situ hybridisation of Prl8a2 at the E7 implantation site to localise PDZ cells for volume analysis. Control (white bars) and PC-EtOH exposed (black) data are shown as mean \pm s.e.m. (A,B) Hormone profiles analysed by two-way ANOVA, $\mathrm{E} 5$ data $n=9 /$ treatment, E6 $n=3$ /treatment, $\mathrm{E} 7 n=3$ /treatment.(C-F) Uterine expression data analysed by two-way ANOVA (overall effects across ages). Because of significant differences in age (E5 versus E7), treatments were analysed at each gestational age with Student's $t$-tests. $n=6 /$ treatment (E5) and $n=10 /$ treatment (E7). Gene expression is relative to control group at $\mathrm{E} 5$, and standardised to the geometric mean of $R p / 19$ and $A c t b$. (G,H) Western blots show $n=5-6 /$ treatment. (J, K) E7 stereology data analysed by Student's $t$-test, $n=4-7 /$ treatment. ${ }^{*} P<0.05$, ${ }^{* *} P<0.01$. Scale bars: $1 \mathrm{~mm}(\mathrm{I}) ; 400 \mu \mathrm{m}(\mathrm{L})$.

PC-EtOH. Invasive trophoblasts were localised by histological staining for the pan-trophoblast marker pan-cytokeratin (Fig. 4B). At E13, when only SpA-TGCs are invasive, placentae from PC-EtOH-exposed females showed reduced invasion depth $(24 \%)$ compared with control females $(P<0.05$, PInt $<0.05$; Fig. 4C). Quantification of SpA-TGC number into uterine spiral arteries showed that PC-EtOH males and females had reduced cell counts $(P \operatorname{Trt}<0.05$; Fig. 4D). Investigation of SpA-TGC DNA content showed an interaction of treatment with sex after PC-EtOH $(P$ Int $<0.01$; Fig. 4E), with PC-EtOH females exhibiting increased DNA content $(P<0.05)$. Assessment of spiral artery remodelling was performed by localisation of alpha smooth muscle actin $(\alpha \mathrm{SMA})$ and showed that PC-EtOH increased $\alpha$ SMA intensity by $9 \%$ in males and $7 \%$ in females, although this was not statistically significant $(P \operatorname{Trt}=0.08$; Fig. 4F). Similarly, cross-sectional area of uterine spiral arteries was reduced by $26 \%$ and $16 \%$ in PC-EtOH males and females but was not statistically significant ( $P=0.96$; Fig. 4G). In PC$\mathrm{EtOH}$ female placentae at E15, average and maximum depth of invasion were decreased by $24 \%$ and $27 \%$, respectively, although this did not reach statistical significance $(P=0.14$ and $P=0.08$, respectively) (Fig. S6A,B). By E20, no differences were found in average or maximum invasion depth, suggesting an overall delay in invasion in placentae of females (Fig. S6C,D).

\section{PC-EtOH causes female-specific structural perturbations in the placenta, resulting in a greater percentage of males}

Our previous study on PC-EtOH showed increased resorptions/ non-viable fetuses and reduced fetal weight at E20 (Gårdebjer et al., 2014). In the present study, we determined at what point during gestation embryonic viability was compromised. A similar number of viable embryos were found at E13 (Table S3). However, an increase in resorptions was found at E15 $(P<0.05)$, with a greater percentage of viable male fetuses $(P=0.05$; Table 1$)$, suggesting reduced female survival. Embryonic and placental weights were unchanged at E13 in both sexes (Table S3) and further investigation into placental biometry at E15 showed no differences between groups or sex for 

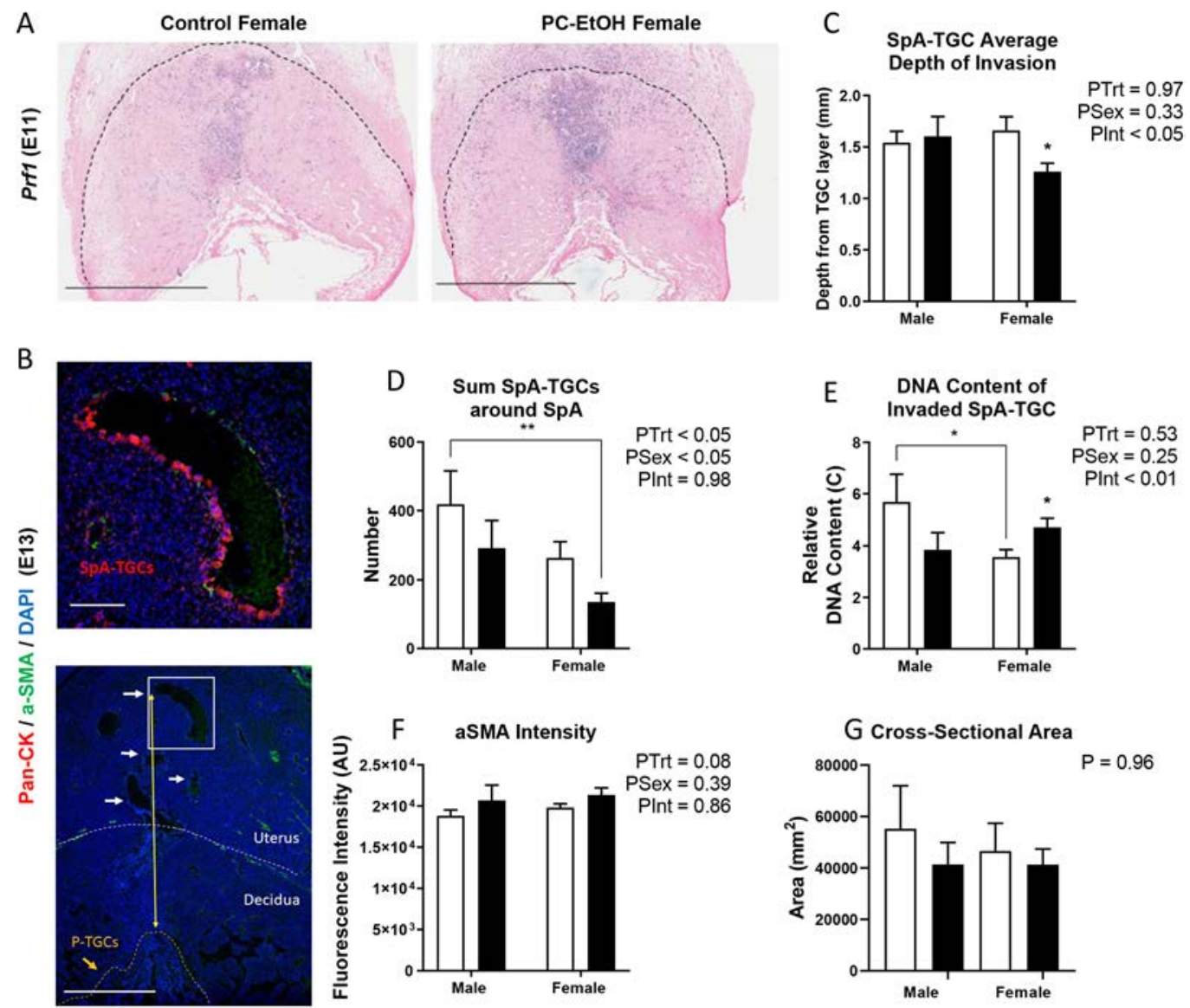

Fig. 4. PC-EtOH exposure alters infiltration of maternal decidual natural killer cells and reduces trophoblast invasion into the mesometrial region in females only. (A) In situ hybridisation for perforin 1 (Prf1) marking dNK cells in control and PC-EtOH-exposed female placentae. Dashed lines show barrier between decidua and uterus. (B) Immunolocalisation of pan-cytokeratin (red) marking trophoblasts and alpha smooth muscle actin (aSMA, green) with DAPI (blue) as counterstain in E13 placenta. White dashed line shows barrier between uterus and decidua. Orange dashed line shows P-TGC border. Upper panel shows higher magnification of the boxed area below. Arrows indicate maternal spiral arteries. (C-G) Invasion was assessed at E13 by measuring the average invasion depth of SpA-TGCs measured from the P-TGC layer (C), the total number of invaded SpA-TGCs around spiral arteries (SpA) (D), the DNA content of invaded SpA-TGCs (E), the intensity of alpha smooth muscle actin (aSMA) (F), which stains of uterine SpA vessels, and the cross-sectional area of uterine SpA (G). Control (white bar), PC-EtOH exposed (black bar) data are shown as mean \pm s.e.m. (C-F) Two-way ANOVA for treatment with sex. (G) Non-parametric Kruskal-Wallis test. (C) $n=11-19 /$ sex from 6-7 litters/treatment. (D,E) $n=6-12$ 5-6 litters/treatment. (F) Pooled data from $n=4-8$ placentae from 2 litters/ treatment. (G) $n=8-11$ placentae from 5-6 litters/treatment. ${ }^{*} P<0.05$, ${ }^{\star *} P<0.01$. Scale bars: $2 \mathrm{~mm}$ (A); $1 \mathrm{~mm}$ (B, top); $200 \mu \mathrm{m}$ (B, bottom).

embryonic or placental weight, placental efficiency ( placental weight to body weight ratio), labyrinth or junctional zone wet weight, or the ratio of zone weight to volume (Table 1). However, reduced placental depth was found after PC-EtOH in both males and females (Table 1), suggesting less vascularisation.

We next investigated the morphogenesis of placental structure in more detail, particularly the labyrinthine vasculature. Using unbiased stereology, we quantified volume and surface area of the placental compartments on the E13 (immature), E15 (definitive) and E20 (mature) placenta. Again, it was E15 that showed significant differences, with E13 and E20 stereology remaining unchanged between treatments (Table S4). At E15, although there was no change to the volumes of the whole placenta, decidua, labyrinth or junctional zone in PC-EtOH males (Fig. 5A), PC-EtOH females showed markedly reduced (by 17\%) placental volume, with all compartments contributing to this overall effect $(P<0.01 ;$ Fig. 5B). Examination of the placental vasculature for fetal and maternal blood spaces (FBS and MBS, respectively) showed the placenta of PC-EtOH females to have significantly reduced MBS volume ( $P<0.05$; Fig. 5D). The fetal vasculature was marked using the ISH probe Mest for fetal endothelial cells (see Fig. 5I,J for representative images of female labyrinths). When MBS volume was analysed with males and females together by two-way ANOVA (treatment with sex), a treatment effect was found after PC-EtOH (PTrt<0.01; Fig. S7), indicating a subtle effect on male PC-EtOH placentae (20\% reduction versus $32 \%$ reduction in females). No change to FBS or MBS surface area was found between treatments for either sex (Fig. 5E,F). Further, in males, no changes to FBS, MBS or labyrinth trophoblast were found after PC-EtOH (Fig. 5C). Despite the alteration in MBS volume in females, the thickness of the interhaemal membrane remained unchanged at both E15 and E20 (Fig. S8). No differences in groups was shown for labyrinth trophoblast volume (Fig. 5D). Considering the sex-specific outcomes in differentiation, placentae from PC-EtOH females were also examined for DNA content for labyrinthine sinusoidal (S-TGCs) lining the MBS vessels, and secondary P-TGCs located at the interface between the junctional zone and the decidua. Interestingly, both S-TGCs and secondary PTGCs showed reduced DNA content $(P<0.0001$; Fig. 5G,H), suggestive of reduced endoreplication and differentiation.

Perturbation of placental morphogenesis was examined next, using gene expression assays to examine the potential influence of altered differentiation of placental cell types in the E13 whole placenta and 
Table 1. Post-mortem fetal and placental biometry at E15

\begin{tabular}{|c|c|c|c|c|c|}
\hline \multirow{2}{*}{$\frac{\text { Variables }}{\text { Litter size (number) }}$} & \multicolumn{2}{|c|}{ Control } & \multicolumn{2}{|c|}{ PC-EtOH } & \multirow{2}{*}{$\begin{array}{l}\text { Statistics } \\
P=0.42\end{array}$} \\
\hline & $16.33 \pm 0.500$ & & $17.000 \pm 0.646$ & & \\
\hline Resorptions (number) & $0.440 \pm 0.294$ & & $1.889 \pm 0.539$ & & $P<0.05$ \\
\hline Sex ratio (M:F) & $0.94 \pm 0.211$ & & $1.41 \pm 0.177$ & & $P=0.11$ \\
\hline Variables & Male control & Male PC-EtOH & Female control & Female PC-EtOH & Statistics \\
\hline \multicolumn{6}{|l|}{ Fetal parameters } \\
\hline Crown-rump length (mm) & $12.405 \pm 0.245$ & $12.601 \pm 0.048$ & $12.226 \pm 0.195$ & $12.142 \pm 0.092$ & $\begin{array}{l}P \text { Trt }=0.74 \\
P \text { Sex }=0.06 \\
P \text { Int }=0.40\end{array}$ \\
\hline \multicolumn{6}{|l|}{ Placental parameters } \\
\hline Placental weight (g) & $0.247 \pm 0.023$ & $0.212 \pm 0.015$ & $0.217 \pm 0.006$ & $0.218 \pm 0.009$ & $\begin{array}{l}P \text { Trt }=0.23 \\
P \text { Sex }=0.41 \\
P \text { Int }=0.22\end{array}$ \\
\hline Junctional zone wet weight (g) & $0.102 \pm 0.008$ & $0.102 \pm 0.007$ & $0.096 \pm 0.006$ & $0.118 \pm 0.008$ & $\begin{array}{l}P \text { Trt }=0.27 \\
P \text { Sex }=0.34 \\
P \text { Int }=0.49\end{array}$ \\
\hline Labyrinth wet weight (g) & $0.086 \pm 0.009$ & $0.078 \pm 0.006$ & $0.094 \pm 0.006$ & $0.075 \pm 0.004$ & $\begin{array}{l}P \text { Trt }=0.06 \\
P \text { Sex }=0.75 \\
\text { Plnt }=0.39\end{array}$ \\
\hline Placental length $(\mathrm{mm})$ & $12.087 \pm 0.352$ & $12.111 \pm 0.212$ & $12.144 \pm 0.232$ & $12.297 \pm 0.155$ & $\begin{array}{l}P \text { Trt }=0.15 \\
P \text { Sex }=0.63 \\
P \text { Int }=0.80\end{array}$ \\
\hline Placental width $(\mathrm{mm})$ & $11.411 \pm 0.245$ & $11.027 \pm 0.233$ & $11.037 \pm 0.187$ & $11.191 \pm 0.209$ & $\begin{array}{l}P \text { Trt }=0.60 \\
P \text { Sex }=0.64 \\
P \text { Int }=0.23\end{array}$ \\
\hline Placental depth (mm) & $2.979 \pm 0.104^{*}$ & $2.590 \pm 0.097$ & $2.836 \pm 0.079^{*}$ & $2.653 \pm 0.083$ & $\begin{array}{l}P \text { Trt }<0.01 \\
P \text { Sex }=0.65 \\
P \text { Int }=0.26\end{array}$ \\
\hline
\end{tabular}

All data are presented as mean \pm s.e.m. $n=4-12 /$ sex from 9 litters/treatment for all data except labyrinth weight:labyrinth volume and junctional zone weight: junctional zone volume, $n=5-8$ from 4-7 litters. Litter size, resorptions, sex ratio and percentage males were analysed by Student's $t$-tests, $n=9$ litters/treatment. Remaining data were analysed by two-way ANOVAs, ${ }^{*} P<0.05$ for Bonferroni post-hoc compared with controls of same sex. Note: litter size includes resorptions. F, female; M, male.

the E15 labyrinth. Mest was used to identify fetal endothelial cells, Syna for syncytiotrophoblast layer 1 cells, Gcml for syncytiotrophoblast layer 2 cells, Cts $q$ for sinusoidal TGCs, and Prl3d1 for secondary P-TGCs (only at E13). Curiously, only Mest was shown to be reduced at E13 ( $P \operatorname{Trt}<0.01$; Fig. S9A), particularly in male PC-EtOH placentae $(P<0.05)$. At E15, statistical interactions were found between treatment and sex for both Mest and Syna, suggesting that males tended to show decreased expression and females increased expression ( $P$ Int $<0.05$ for both genes; Fig. S9F,I). No changes were found in other markers at either age.

\section{PC-EtOH alters expression of labyrinthine glucose transporters and reduces placental clearance of [ $\left.{ }^{3} \mathrm{H}\right]$-glucose}

To determine whether altered placental morphology was associated with altered nutrient transfer after $\mathrm{PC}-\mathrm{EtOH}$, gene expression assays for glucose transporters [Glut1 (Slc2a1), Glut3 (Slc2a3)] and system A amino acid transporters (Slc38a1, Slc38a2, Slc38a4) were conducted at E13 and E15. At E13, Glut3 expression was significantly decreased $(P \operatorname{Trt}<0.01$; Fig. $6 \mathrm{~B})$, particularly in females $(P<0.05)$, with no apparent changes in Glutl (Fig. 6A). Interestingly, by E15, an increase was found in Glut1 after PC-EtOH $(P$ Trt $<0.05$; Fig. $6 \mathrm{C})$, particularly in females $(P<0.05)$, and Glut3 showed an interaction ( $P$ Int $<0.05$; Fig. 6D), suggesting that male PC-EtOH placentae had decreased expression, and female PC-EtOH placentae had increased expression. No changes to system A amino acid transporters were found at either gestational age (Fig. S10).

To determine the capacity of the PC-EtOH-exposed placenta to transport glucose and supply fetal growth, a non-metabolised $\left[{ }^{3} \mathrm{H}\right]$-O-methyl-D-glucose (H3G) tracer was injected into dams at E13 and E15. Absolute fetal accumulation of the radiotracer, placental transport, placental uptake, and placental clearance relative to maternal blood levels were calculated. At E15, PC-EtOH exposure caused a reduction of fetal accumulation (Fig. 6E), relative transport (Fig. 6F), placental uptake (Fig. 6G) 

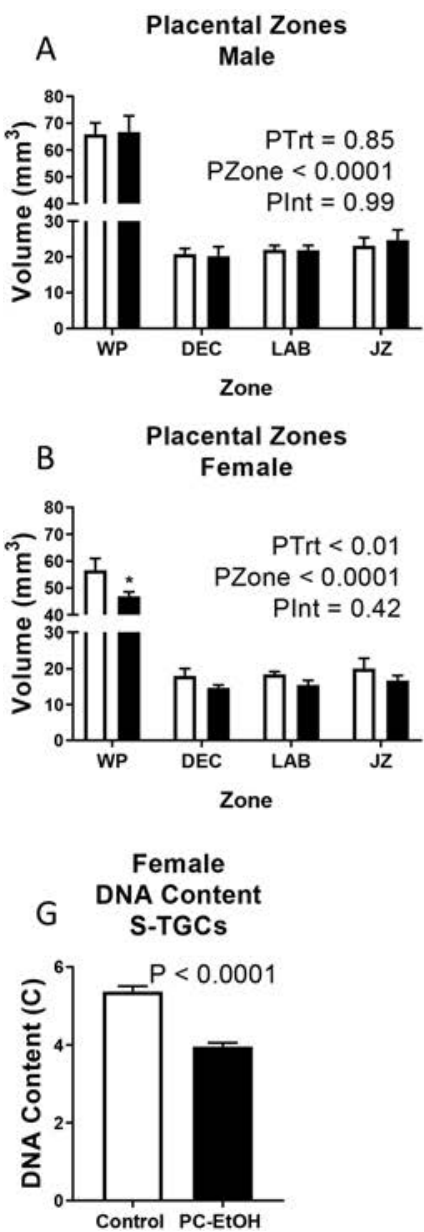

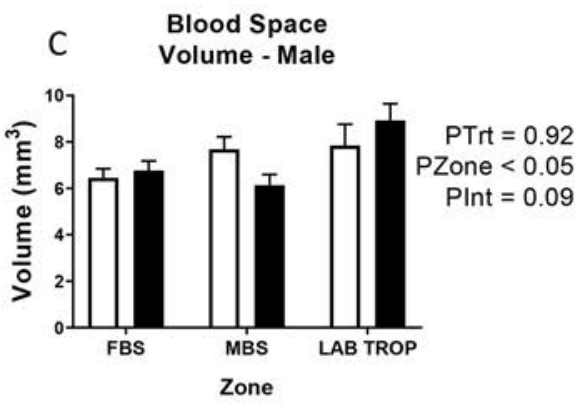

Blood Space

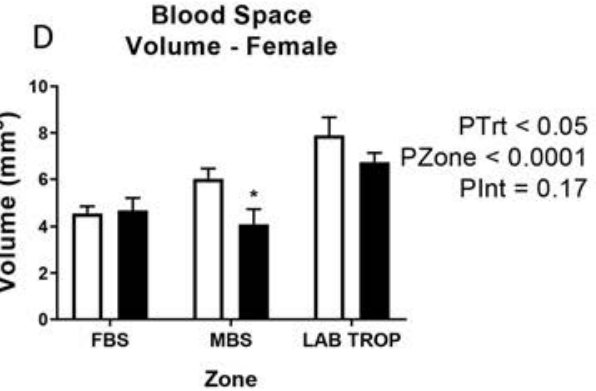

B Blood Space

F Surface Area - Female

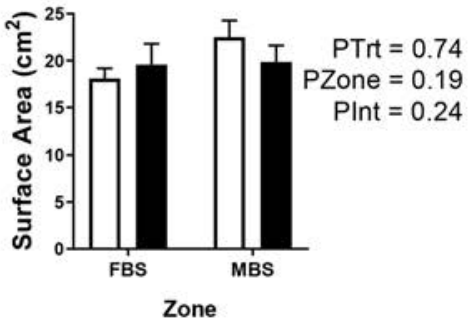

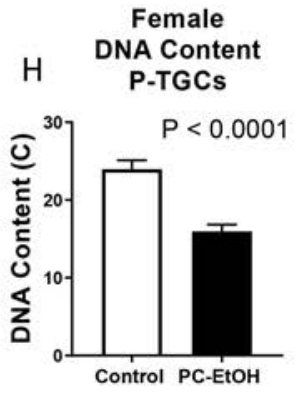
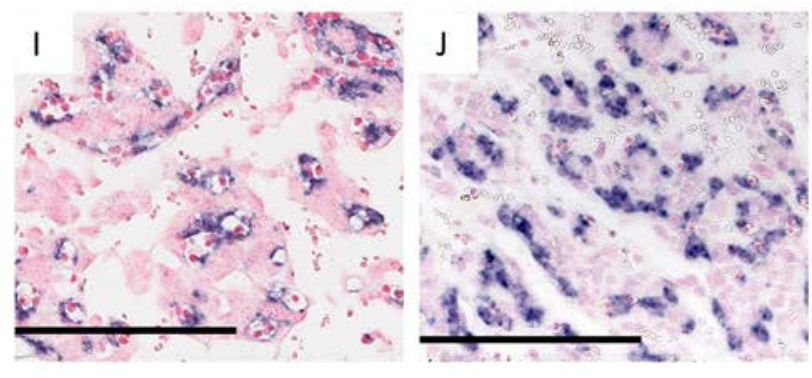

Fig. 5. PC-EtOH exposure causes female-specific alterations to placental volumes, maternal blood space vascularisation and differentiation of trophoblast giant cells. (A,B) Volumes of whole placenta (WP), decidua (DEC), labyrinth (LAB) and junctional zone (JZ) in E15 males (A) and females (B). $(C, D)$ Volume of labyrinthine fetal (FBS) and maternal (MBS) blood spaces and labyrinthine trophoblast (LAB TROP) in males (C) and females (D).

$(E, F)$ Surface area of labyrinth FBS and MBS in males $(E)$ and females $(F) .(G, H)$ Female MBS spaces were further evaluated for DNA content of sinusoidal trophoblast giant cells (S-TGCs; G) and secondary parietal trophoblast giant cells (P-TGCs; H). (I,J) Representative images of female placental labyrinths marked with ISH probe Mest, for control (I) and PC-EtOH (J). Control (white bars), PC-EtOH exposed (black bars) data are shown as mean \pm s.e.m. Note: two-way ANOVA of MBS volume (treatment versus sex) shows $P$ Trt $<0.01$ (see Fig. S3). (A-F) Two-way ANOVA. (G,H) Student's $t$-test was used for DNA content. * $P<0.05$. (A-F) $n=7-9 /$ sex, 5-7 litters/treatment. (G,H) DNA content of TGCs were standardised to decidual cells $(2 \mathrm{~N})$ of the same placenta and pooled from $n=7-8$ placentae from 5-6 litters/treatment, giving 299-360 measurements for S-TGCs (G) and 170-182 measurements for secondary P-TGCs (H). All data taken from E15 samples. ${ }^{*} P<0.05$. Scale bars: $200 \mu \mathrm{m}$

and placental clearance (Fig. 6H) of H3G. A basal sex difference was identified in placental clearance, which showed that control females cleared less $\mathrm{H} 3 \mathrm{G}$ than control males $(P<0.05)$. No changes to E13 parameters were shown after PC-EtOH (Fig. S11).

\section{PC-EtOH alters expression of imprinted and X chromosome inactivation genes}

A recent study of human placentae has associated aberrant expression of imprinted genes with diagnosis of early-onset pre-eclampsia and phenotypes of poor trophoblast proliferation and invasion (Zadora et al., 2017). To determine whether perturbed imprinting may also be occurring after PC-EtOH, as a possible longterm consequence of perturbed epigenetic reprogramming from the pre-implantation stage, selected genes were assayed in the E13 whole placenta. Expression of maternally expressed (paternally imprinted and silenced) genes (Igf $2 r$, Phlda2, Cited1) and paternally expressed genes (Igf2) were assessed (Fig. 7). Igf2r expression was decreased after PC-EtOH in both sexes $(P \operatorname{Trt}<0.01$; Fig. 7B), but the decrease was greater in females (40\% reduction) than males (31\% reduction). Phlda2 was similarly decreased in both sexes after PC-EtOH $(P<0.05$; Fig. $7 \mathrm{C})$, predominantly owing to reduced expression in the placentae of females. Cited 1 and paternally expressed $I g f 2$ were unchanged (Fig. 7A,G), whereas paternally expressed Mest decreased after PC-EtOH, particularly in males $(P \operatorname{Trt}<0.01, P$ Male $<0.05$; Fig. 7A).

Known X-linked genes, G6pd, Hprt (Hprt1), Xist, Cited1 (see above), as well as those involved in imprinted $\mathrm{X}$ chromosome inactivation (XCI), Xist and Rlim, were examined after PC-EtOH. $\mathrm{XCI}$ in female TE and ICM cells ensures appropriate gene dosage between males and females. XCI is also unique in trophoblast cells compared with other somatic lineages, which use random XCI, as the paternal $\mathrm{X}$ chromosome (Xp) is exclusively inactivated (and thereby 'imprinted') (Senner et al., 2011). Female primary and secondary TGCs are exceptional in that they are able to partially reexpress genes on this 'inactive' Xp (Hadjantonakis et al., 2001; Corbel et al., 2013). Expression of G6pd was found to be decreased after PC-EtOH in both sexes $(P \operatorname{Trt}<0.0001, P$ Male $<0.05$, $P$ Female $<0.001$; Fig. 7D). Curiously, expression of Hprt was 

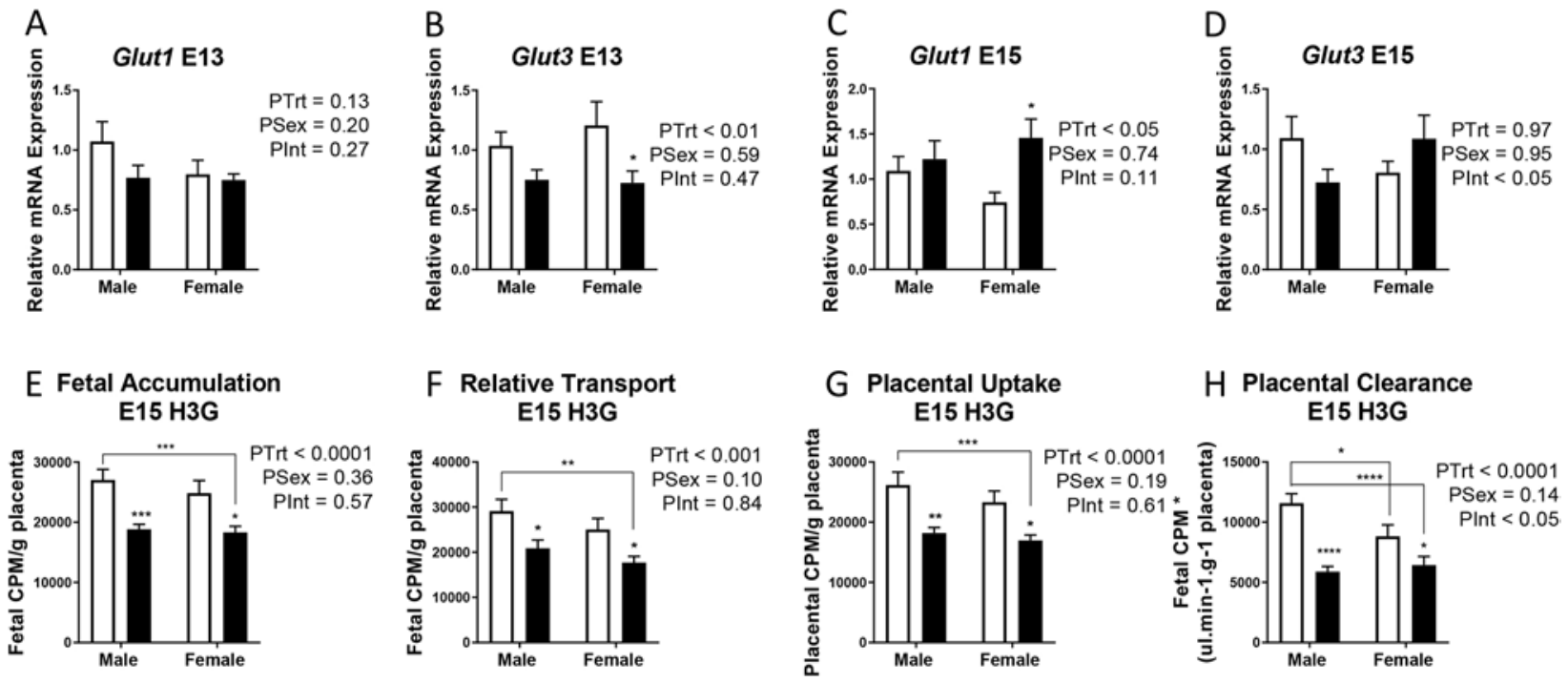

Fig. 6. PC-EtOH exposure causes sex-specific alterations to placental glucose transport over gestation. (A-D) Expression of nutrient transporters were assessed in the E13 whole placenta and E15 labyrinth. Glucose transporters Glut1 (SynT-1 facing MBS side, SynT-II facing FEC, and JZ) and Glut3 (SynT-1 facing MBS side) were assessed at E13 (A,B) and E15 (C,D). Expression data are relative to the geometric mean of 18S and Rp/13a, and standardised to average expression of the control male group for each age. $(\mathrm{E}-\mathrm{H})$ Total glucose was assessed using the glucose analogue $\left[{ }^{3} \mathrm{H}\right]-\mathrm{O}-\mathrm{methyl-D-glucose}(\mathrm{H} 3 \mathrm{G})$ at E15. Assays assessed fetal accumulation (fetal CPM $/ \mathrm{g}$ fetus), relative placental transport (fetal CPM $/ \mathrm{g}$ placenta), placental uptake ( $\mathrm{placental}$ CPM $/ \mathrm{g}$ placenta) and placental clearance (fetal CPM * $\mu \mathrm{min}^{-1} \mathrm{~g}^{-1}$ placenta). Control (white bars), PC-EtOH exposed (black bars) data are shown as mean \pm s.e.m. and analysed by two-way ANOVA. ${ }^{*} P<0.05,{ }^{* *} P<0.01$, ${ }^{* * *} P<0.001,{ }^{* * *} P<0.0001$ from Bonferroni post-hoc tests. (A-D) E13: $n=7-8 /$ sex from 4-5 litters/treatment; E15: $n=7-8 /$ sex from $7-8$ litters/treatment. $(E-H) n=18-23$ from 3 litters per treatment.

increased after PC-EtOH $(P<0.01, P$ Male $<0.01 ;$ Fig. 7 F $)$. When female placentae were examined for XCI initiation, expression of both Xist and Rlim (also known as Rnf12) were both significantly decreased after $\mathrm{PC}-\mathrm{EtOH}(P<0.001$ and $P<0.05$, respectively; Fig. 7H,I). Expression of the sex-specific stress-mediated enzyme Ogt was also analysed, as it is known to escape XCI in females, but was unchanged after PC-EtOH (Fig. 7E).

\section{PC-EtOH exposure and a potential epigenetic link to later development}

Previous investigation into a direct impact of alcohol on mouse trophoblast stem cells showed downregulation of the expression of mature trophoblast lineage markers at day 6 of differentiation (Kalisch-Smith et al., 2016). Here, we continued to investigate an earlier time point of day 2 of differentiation and showed no change in gene expression for $C d x 2$ (Fig. S12). This suggests that the impacts of PC-EtOH are more likely to be due to an indirect mechanism, such as through an altered uterine milieu. To investigate this further, we characterised maternal plasma choline levels over the pre-implantation period. Choline is part of the one-carbon metabolism pathway, which can be a source of methyl donors and thereby facilitate normal methylation patterning (reviewed by Zeisel and da Costa, 2009). Maternal choline was assessed at E2 and E5 (Fig. 8A), and was shown to be reduced by PC-EtOH over this period $(P \operatorname{Trt}<0.05)$. Maternal choline levels also increased from E2 to E5 $(P$ Age $<0.001)$, suggesting an increased demand of choline during pre-implantation development. Maternal triglycerides were also found to be increased after PC-EtOH at E5 (Fig. 8B). Ethanol consumption is often associated with fat accumulation in the liver, and its removal is reliant on a choline-dependent pathway (Zeisel, 2006). Endogenous choline is synthesised in the liver and can be converted from phospholipids through the enzyme Pemt (Zeisel, 2006). The uterus has a limited capacity to synthesise choline compared with the liver (Fig. S13B). Liver expression of Pemt expression was unchanged after PC-EtOH (Fig. 8D). Choline can also be converted to betaine, which is a methyl donor in the production of methionine from homocysteine using the enzyme Bhmt (Barak et al., 1996). Because Bhmt is not expressed by the preimplantation embryo (Kwong, et al., 2010), it must rely on methyl groups from another source, for example the liver or uterus. Similarly to Pemt, Bhmt was also found to be minimally expressed by the uterus (Fig. S13A). Liver Bhmt expression was also unchanged (Fig. 8C). Not only does this suggest that the uterus has little capacity to provide methyl donors through the choline pathway as shown above, it suggests that the embryo must rely on histotrophic nutrition via the maternal vasculature as it does for other nutrient-associated pathways (Burton, et al., 2002).

We therefore assessed choline and methionine transporters in the uterus at E5. The choline transporters Ctll (Slc44al) and Ctl2 (Slc44a2) were unchanged by PC-EtOH (Fig. 8F,G). However, the neutral amino acid transporter Slc38a4, which also transports methionine, was markedly reduced after PC-EtOH $(P<0.01$; Fig. 8E). Reduced methionine transport and availability may partially explain the perturbed methylation patterns after PC$\mathrm{EtOH}$, as embryos cannot undergo normal re-methylation and imprinting during reprogramming.

To connect the phenotypes seen in early and late gestation, DNA methylation was assayed in E13 whole placenta, as well as E15 and E20 labyrinth and junctional zone (Fig. 8H-L). Methylation patterns were found to be dynamic, showing progressively reduced DNA methylation from E13 to E20. From this analysis, only the E20 PCEtOH labyrinth showed a significant change, exhibiting marked increases in methylation $(P \operatorname{Trt}<0.01$; Fig. $8 \mathrm{~L})$, particularly in males $(P$ Male $<0.05)$.

\section{DISCUSSION}

This study aimed to investigate the impact of PC-EtOH exposure on developmental processes that may contribute to the established phenotypes of FGR and metabolic dysfunction in this model (Gårdebjer et al., 2014, 2015). Specifically, we sought to examine 

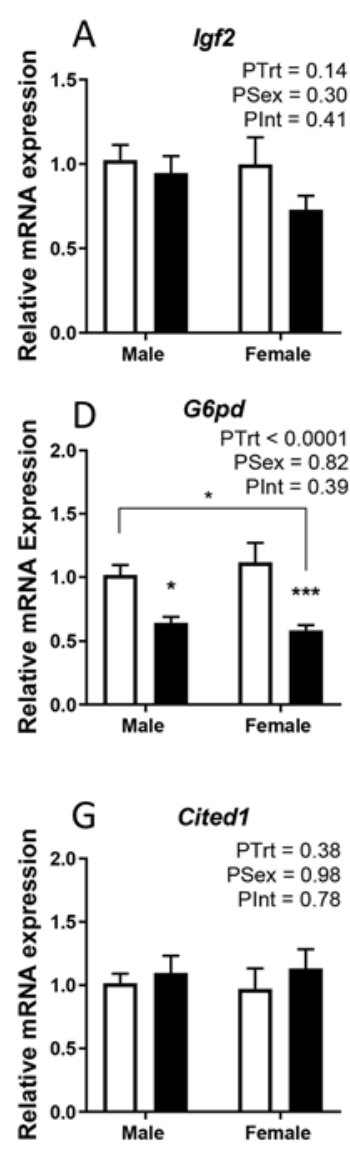
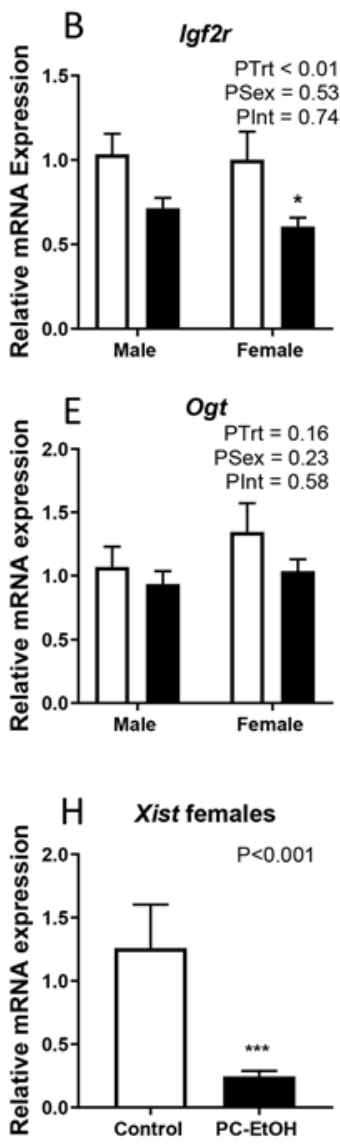

the impact on cell allocation during the primary differentiation of blastocyst formation and the subsequent development of the functional placenta. Moreover, given that epigenetic reprogramming also occurs as a part of pre-implantation development in preparation for embryonic genome activation, we sought to establish whether there were potential changes to DNA methylation in PC-ETOH-exposed embryos. We also wanted to determine if there is a mechanistic link through a direct or indirect (i.e. uterine) impact of ethanol.

In this ontogeny study, we show a sexually dimorphic impact in response to periconceptional alcohol exposure, with primarily a female-biased trophoblast defect (see Fig. 9 for a comprehensive summary). This manifested as perturbed differentiation of multiple TGC subtypes, and, consequently, reduced invasion and vascularisation of the mid-gestation placenta. These defects in females explain the reduced embryonic viability and the increased percentage of male embryos in mid-gestation. Although placentae of males show some of the same outcomes, such as reduced expression of CDX2 (a TE lineage-associated gene), increased $5 \mathrm{mC}$ expression, and trophoblast proliferation, they appear to be affected to a lesser extent. Collectively, this challenges the current dogma that male offspring are more at risk of developmental perturbations due to placental deficits, and rather suggests that there are two critical windows of susceptibility during gestation, affecting males and females differentially, as we have previously proposed (Kalisch-Smith et al., 2017a). We also highlight that a femalespecific trophoblast invasion defect can be associated with perturbed imprinting, epigenetic reprogramming and $\mathrm{X}$ chromosome inactivation. In addition, we provide a mechanistic link to the one-carbon metabolism pathway, whereby reduced maternal choline and methionine transport to the uterus could

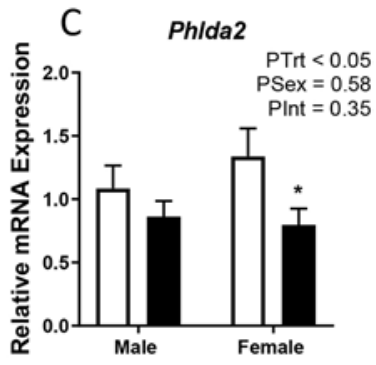

Fig. 7. Gene expression profiles of selected imprinted and $\mathrm{XCl}$ genes on E13 whole placentas after PC-EtOH exposure. (A-I) RT-QPCR profiles of the paternally expressed (maternally imprinted) gene lgf2, and the maternally expressed genes Igf2r, Phlda2 and Cited1, along with X-linked genes (Cited1, G6pd, Hprt and Xist), genes involved in X chromosome inactivation (XCI) (Xist and Rlim), and Ogt, a gene known to escape $\mathrm{XCl}$ in females. Control (white bars) and PC-EtOH exposed (black bars) data are shown as mean \pm s.e.m. and analysed by two-way ANOVA. Expression data are relative to the geometric mean of $18 S$ and Rp/13a, and standardised to average expression of the control male group for each age. $n=7-8 /$ sex from 4-5 litters/treatment. ${ }^{\star} P<0.05,{ }^{* *} P<0.01$, *** $P<0.001$

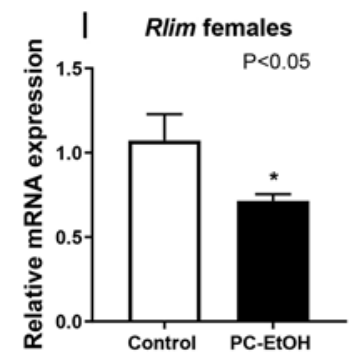

explain a reduced capacity of the embryo to undergo de novo methylation. This study thereby provides a mechanism by which other pregnancy disorders, such as pre-eclampsia, may develop the adverse phenotypes of trophoblast differentiation and invasion (Zadora et al., 2017; reviewed by Burton and Jauniaux, 2018). Recent findings from human early-onset pre-eclampsia (prior to 34 weeks gestation), has also reinforced these findings, showing greater prevalence in female fetuses (Taylor et al., 2018).

Our observations of sexually dimorphic placental outcomes are supported by studies in other models of programming associated with in vitro fertilisation (IVF)- and intracytoplasmic sperm injection (ICSI)-derived mouse embryos (Tan et al., 2016a,b), which show female-specific effects on trophoblast differentiation and placental morphogenesis. Interestingly, genetic mutants of several X-linked genes, Eed (Wang et al., 2001), SmcHD1 (Blewitt et al., 2008), Dnmt1 (McGraw et al., 2013) and Xist (Hoki et al., 2011; Senner et al., 2011; Mugford et al., 2012), all phenocopy the aforementioned maternal nutrient-perturbation models, exhibiting female-specific defects in TGC differentiation. These mutants are associated with altered X-chromosome dosage, pertaining to roles in either XCI initiation through Xist expression, or maintenance by recruitment of the PRC2 complex. Furthermore, female IVF preimplantation embryos at the morula stage have shown reduced expression of Xist and Rlim (Tan et al., 2016a), two initiators of XCI. Although we did not investigate XCI in the pre-implantation embryo, we examined the mid-gestation placenta to determine potential long-term consequences of a hypermethylated genome. Although we did not find evidence of hypermethylation at this stage, similar to the IVF study, investigation of PC-EtOH showed reductions in Xist and Rlim expression in females, suggesting that $\mathrm{XCI}$ is also perturbed in this model. In female embryos, aberrant 

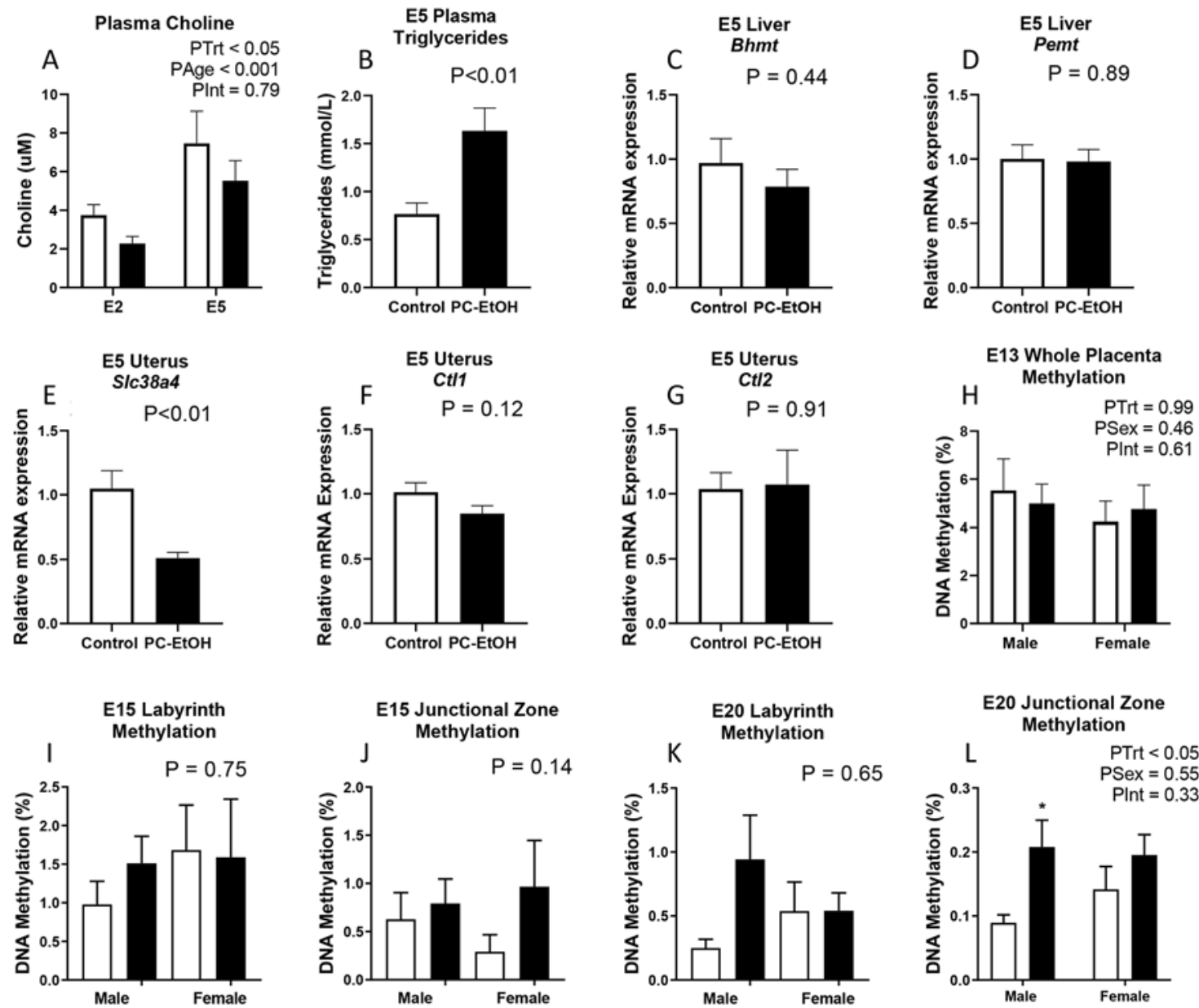

Fig. 8. PC-EtOH exposure reduces maternal choline levels and expression of a uterine methionine transporter and impacts methylation of the junctional zone. (A) Plasma choline levels at E2 ( $n=3 /$ treatment of pooled data over $24 \mathrm{~h}$ ) or E5 ( $n=3 /$ treatment). (B) Plasma triglyceride levels at $\mathrm{E} 5 n=9-10 /$ treatment. (C-G) Gene expression assays (RT-QPCR) in E5 liver (C,D) and E5 uterus (E-G). Expression is relative to control group at E5, and standardised to geometric mean of $R p / 19$ and $A c t b . n=6 /$ treatment (E5 uterus) and $n=8 /$ treatment (E5 liver) from $6-8$ litters per treatment. (H-L) For methylation assays, $n=6-8 /$ sex from 6-8 litters/treatment. ${ }^{*} P<0.05$. Control (white bars) and PC-EtOH exposed (black bars) data are presented as mean \pm s.e.m. (A,L,H) Two-way ANOVA. (B-D,F,G) Student's $t$-tests. (E) Non-parametric Mann-Whitney. (I-K) Non-parametric Kruskal-Wallis tests.

$\mathrm{XCI}$ and consequently inappropriate gene dosage, may be a state of genetic instability, causing them to be particularly sensitive to environmental perturbations during the period of epigenetic reprogramming. In this study, gene assays for Xist and Rlim were investigated in the E13 whole placenta, a heterogeneous cell population deriving from maternal, placental and fetal-derived tissues. However, considering the major structural and differentiative changes found in the multiple TGC subtypes after PC-EtOH, over that of all of the other trophoblast subtypes investigated, it is likely that these TGCs are primarily driving this aberrant XCI phenotype.

Environmental perturbation models can cause heritable changes in cellular memory, such as through DNA methylation, and thereby contribute to developmental programming (Ozanne and Constância, 2007; Sandovici et al., 2012). During pre-implantation development, maternally derived nutrient substrates in the one-carbon metabolism pathway, such as folate, choline, methionine and betaine, act as methyl donors to produce S-adenosylmethionine (Kwong, et al., 2010). This is the primary substrate for the DNMT enzymes, which are required to facilitate de novo methylation from the blastocyst stage. If these nutrients are either in deficiency or excess during this sensitive pre-implantation period, they can lead to aberrant methylation patterns and impact developmental competence
(O’Neill, 1998; Kwong, et al., 2010, Sinclair et al., 2007; Padmanabhan, et al., 2013). Human studies also report perturbed methylation patterns and altered one-carbon metabolites in adverse environments, including alcohol exposure (Refsum et al., 2006; Steegers-Theunissen, et al., 2013). Indeed, alcohol has been shown to affect several parts of the one-carbon metabolism pathway and impact on DNA methylation (reviewed by Kobor and Weinberg, 2011; Ouko et al., 2009). We show maternal choline can be reduced after PC-EtOH, but ethanol can also impact folate transporter function and folate uptake (Hamid et al., 2007; Halsted et al., 2002; Naughton et al., 1989). Ethanol can reduce methionine synthase activity, the enzyme that converts homocysteine to S-adenosylmethionine (Barak, et al., 1996), and alters expression of the DNMT enzymes (Garro et al., 1991; Bielawski et al., 2002), thereby affecting DNA methylation. The long-term effects of perturbed methylation include those impacting genomic imprinting, as seen here after PC-EtOH, or by others (Haycock and Ramsay, 2009), and those impacting pre- and post-natal tissues (Kaminen-Ahola, et al., 2010). Additionally, the importance of one-carbon metabolites is highlighted in experiments showing that choline supplementation can prevent some deleterious effects of ethanol (Otero et al., 2012).

Although aberrant methylation patterns in imprinting, and perhaps $\mathrm{XCI}$, might be explained by reduced methyl donor availability from 


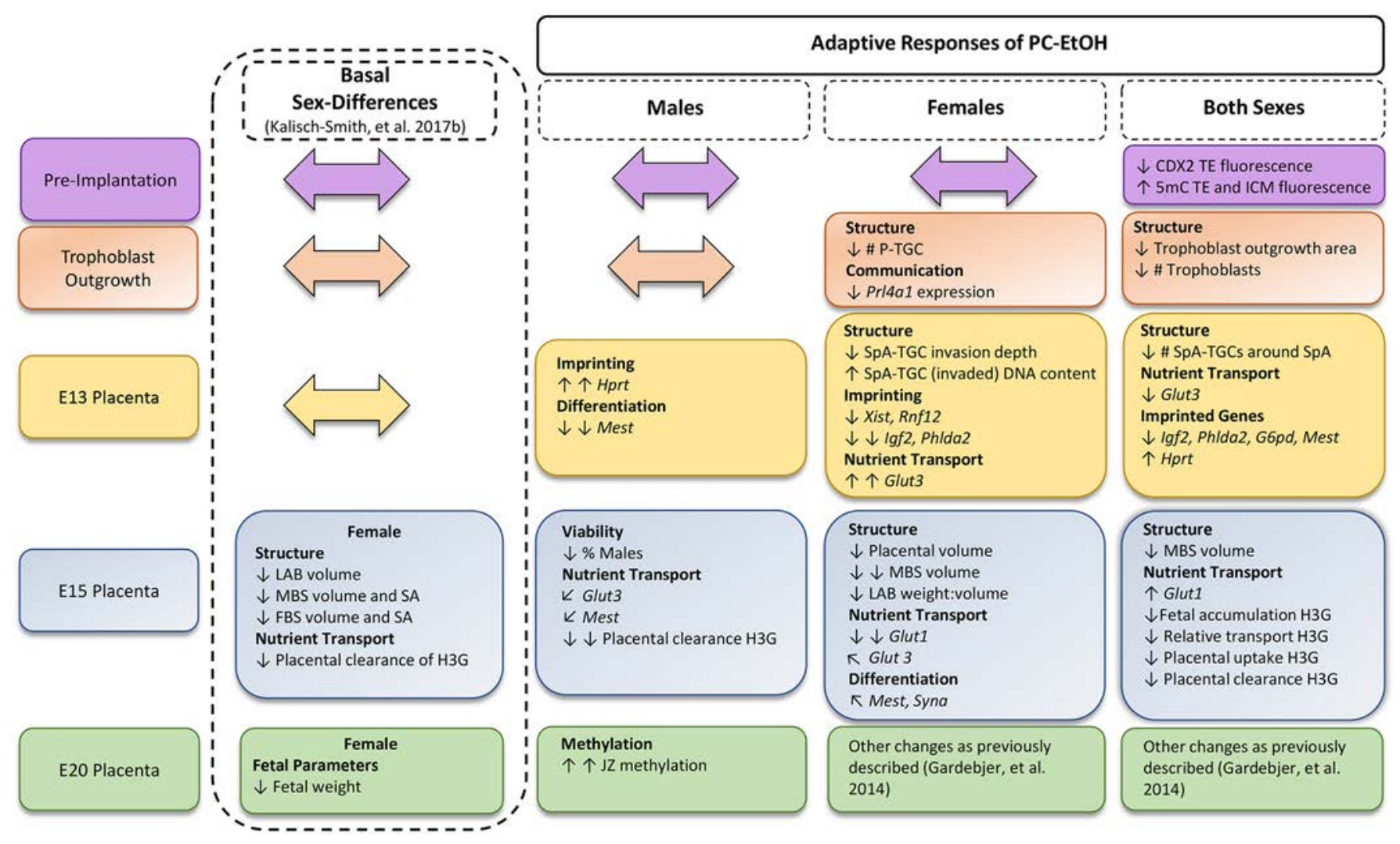

Fig. 9. Summary of basal and sex-specific outcomes during development and after PC-EtOH exposure. Basal sex differences are taken from previously published data (Kalisch-Smith et al., 2017b). Large horizontal arrows indicate no change; diagonal arrows indicate that the post-hoc test revealed an interaction. FBS; fetal blood space, H3G; [ $\left.{ }^{3} \mathrm{H}\right]-\mathrm{O}-$ methyl-D-glucose, ICM; inner cell mass, JZ; junctional zone, LAB; labyrinth, MBS; maternal blood space, $\mathrm{SA}$; surface area, SpA; spiral artery, TE; trophectoderm.

the blastocyst stage, they do not explain why DNA methylation is increased in blastocysts after PC-EtOH. During epigenetic reprogramming, maternal and paternal methylation marks are removed to initiate activation of the embryo's de novo genome. This process involves both untargeted (passive) global demethylation, as well as targeted (active) demethylation. The latter recruits the ten-eleven translocation (TET) protein family to catalyse oxidation of $5 \mathrm{mC}$ methylation marks to $5 \mathrm{hmC}$, which is required for expression of genes involved in the establishment of cell identity (e.g. pluripotency factors) (Hore et al., 2016; Milagre et al., 2017). Interestingly, TET enzyme expression, particularly TET2, is enhanced by retinoic acid (RA) in embryonic stem cells, and thereby accelerates reprogramming (Hore et al., 2016). It is well established that ethanol can compete with enzymes involved in RA biosynthesis, such as ADH and ALDH, which are expressed widely in oviductal and uterine tissues (reviewed by Kalisch-Smith and Moritz, 2017), and is often associated with decreases in RA levels in maternal blood and presumably oviductal fluid. Furthermore, the TET enzymes have recently been shown in trophoblast stem cells to be involved in self-renewal with Tet1/2 mutants showing induction into TGCs (Chrysanthou et al., 2018). Considering that PC-EtOH exposure caused hypermethylation of $5 \mathrm{mC}$, and no change to $5 \mathrm{hmC}$, we hypothesise that reduced RA synthesis in the oviduct and uterus could lead to lower TET activation, causing inappropriate reprogramming through retention of $5 \mathrm{mC}$ methylation marks. This may also explain why PC-EtOH did not result in sexual dimorphism in CDX2 expression, as it is a chief transcription factor involved in trophoblast stem cell identity, which are not classically thought to show sexual dimorphism during establishment of pluripotent lineages
(Milagre et al., 2017). Furthermore, RA has been shown to promote TGC differentiation (Yan et al., 2001) and ameliorate the femalespecific trophoblast XCI defect after IVF in pre-implantation embryos via upregulation of Rlim and Xist expression. Although the details of this process are yet to be elucidated, recent data from Milagre et al. (2017) have shown female mouse embryonic fibroblasts (MEFs) exhibit greater global untargeted demethylation during reprogramming to embryonic stem cells, a similar process to that which occurs in pre-implantation development. Reprogramming of MEFs also involves reactivation of the inactive $\mathrm{X}$ chromosome and could therefore be due to a similar gene dosage effect.

Two other major findings of this study were reduced expansion of the MBS compartment, most significantly in females, and reduced nutrient exchange to both male and female fetuses. This likely explains the late-gestation fetal growth restriction in both sexes of this model (Gårdebjer et al., 2014). A reduction in MBS expansion after PC-EtOH is likely to be caused by reduced perfusion of maternal blood from constricted spiral arteries as a result of delayed invasion and remodelling. Indeed, reduced placental blood flow has already been shown in a first trimester model of alcohol exposure in the rhesus macaque (Lo et al., 2017). A maternal systemic effect may explain why male PC-EtOH conceptuses also show reduced MBS volume, albeit not as severely as the PC-EtOH females. These results also suggest a role of the spiral arteries and maternal blood flow in expanding the MBS of the labyrinth. Reduced vascularisation of only the MBS compartment may be unique to early life alcohol exposure, as other models, such as hypoxia (Higgins et al., 2016), undernutrition (Coan et al., 2010), and a highfat high-sugar diet (Sferruzzi-Perri et al., 2013), additionally show 
reductions in the FBS compartment. Moreover, active transport of $\left[{ }^{3} \mathrm{H}\right]$-glucose showed reduced exchange to the fetus and placental clearance. This coincided with altered Glut transporter expression in the labyrinth, with males showing reduced expression of Glut3, and females showing increases in both Glut1 and Glut3. Both transporters happen to be localised on SynT-1 facing the MBS side and connected to the S-TGCs, which may be a reflection, at least in females, of a reduced surface area of the fenestrated S-TGCs to provide SynT-1 cells with direct contact with maternal blood, and therefore nutrients (Coan et al., 2005). Interestingly, structural changes in the placenta were no longer apparent in the E20 placenta, suggesting that the labyrinth has the capacity to compensate for these structural defects, similar to other programming models (Sferruzzi-Perri et al., 2013; Coan et al., 2010).

\section{Conclusions}

This study provides significant mechanistic insight into the origins of placental defects, fetal growth, and development of adult disease from a perturbed in utero environment around the time of conception. Here, we also provide evidence of unique sex-specific outcomes in response to alcohol exposure that persist through gestation. This model also broadens our understanding of trophoblast-invasion defects, which have not been previously implicated in models of altered maternal diet. Furthermore, our results demonstrate the need for a greater public health focus on the drinking habits of women prior to and around conception.

\section{MATERIALS AND METHODS Ethics}

All animal experiments and procedures were approved by The University of Queensland Animal Ethics Committee (AEC approval number SBS/022/12/ NHMRC) prior to commencement of this study.

\section{Animal treatment, embryo collection and culture}

PC-EtOH exposure was administered in vivo to Sprague Dawley dams in a liquid diet containing $12.5 \% \mathrm{v} / \mathrm{v}$ ethanol as previously described (Gårdebjer et al., 2014, 2015). Dam weight was at least $230 \mathrm{~g}$ before entering the protocol. Vaginal impedance was measured daily with an EC40 oestrous cycle monitor (Fine Science Tools), with $4.5 \times 10^{3} \Omega$ or higher indicating oestrus and allocation to the diet regime. Dams were treated over two oestrus cycles, one prior to mating and the establishment of the pre-implantation period (denoted as E-4 to E4). Dams were time-mated at E0 from $12.00 \mathrm{~h}$ to $17.00 \mathrm{~h}$ if impedance exceeded $4.5 \times 10^{3} \Omega$. If pregnancy was established, the following day was considered E1. At $09.00 \mathrm{~h}$ on E5, dams ( $n=16-18$ /group) were sacrificed via guillotine prior to dissection of the uterine horns and embryo collection. Oviducts and uteri were flushed in HEPES-KSOM media (Lawitts and Biggers, 1993) and allocated to either staining or culture procedures. All embryos were genotyped for sex as previously described (Kalisch-Smith et al., 2017b).

\section{Trophoblast outgrowth assays}

Assays were carried out as previously described (Kalisch-Smith et al., 2017b). A subset of in vivo-treated embryos at E5 were allocated to trophoblast outgrowth assays. Embryos were placed into preheated microdrops of trophoblast stem cell media on tissue culture plates (for subsequent QPCR) or onto gelatine-coated coverslips (for immunostaining), and cultured for 6 days in a humidifying chamber (Minc benchtop incubator, K-MINC-1000, Cook Medical, Australia) at $37^{\circ} \mathrm{C}, 5 \% \mathrm{CO}_{2} / 5 \% \mathrm{O}_{2} / 90 \% \mathrm{~N}_{2}$ under paraffin oil. Media was changed every 2 days and imaged with an inverted phase contrast microscope (Leica). The area occupied by outgrowths were traced in ImageJ using calibrated settings.

\section{Immunofluorescence procedures}

Immunolocalisation for embryos and trophoblast outgrowths was carried out as previously described (Pantaleon et al., 2010; Kalisch-Smith et al., 2017b). Briefly, tissues were fixed in $2 \%$ paraformaldehyde (PFA) in $1 \times$ PBS for $20 \mathrm{~min}$ at room temperature (RT), washed in PBS, and immobilized on Cell-Tak (Collaborative Biomedical Products)-coated coverslips (see Fig. S14). E13 placentae were fixed overnight in 4\% PFA prior to hemisectioning and processing into cryomoulds. Placental halves were embedded cut-face down, and were sectioned at $10 \mu \mathrm{m}$, with every 10th section used for staining. Tissues were permeabilised in $0.25 \%$ Triton X-100 in PBS and washed before blocking with $10 \%$ normal goat serum $/ 4 \mathrm{~g} / 1 \mathrm{BSA} / 0.01 \%$ Tween-20/PBS (PBT) for $1 \mathrm{~h}$ RT. Primary antibodies were incubated overnight at $4^{\circ} \mathrm{C}$. Anti-mouse $\mathrm{CDX} 2$ (BiogenX, MU392A-UC; 1:50) was used to localise TE cells of preimplantation embryos, whereas trophoblast outgrowths and E13 placentae utilised anti-rabbit pan-cytokeratin (DAKO, Z0622; 1:200) to distinguish the cytoskeleton of trophoblasts, and anti-mouse alpha smooth muscle actin (ab7817, Abcam; 1:100) to distinguish uterine spiral arteries and vascular smooth muscle cells. Methylation studies were carried out with specific permeabilisation $(0.5 \% \mathrm{v} / \mathrm{v}$ Tween 20 and Triton X-100 in PBS for $40 \mathrm{~min}$ at $\mathrm{RT}), \mathrm{HCl}(4 \mathrm{~N} \mathrm{HCl} 10 \mathrm{~min})$ and tryptic digestion $(0.25 \% \mathrm{w} /$ v trypsin at $37^{\circ} \mathrm{C}$ for $30 \mathrm{~s}$ ) steps and an overnight blocking step in $30 \%$ normal goat serum as described by $\mathrm{Li}$ et al. (2016), using anti-mouse $5 \mathrm{mC}$ (AbD Serotec, Raleigh, NC, Clone 33D3; 1:100) and anti-rabbit 5hmC (Active Motif, 39769; 1:150). The following day, tissues were extensively washed in PBT prior to incubation with goat anti-mouse Alexa 488 (Molecular Probes, A-11001), goat anti-rabbit Alexa 568 (Molecular Probes, A-11011) or goat anti-rabbit Cy3 (Molecular Probes, A-21428) secondary antibodies at 1:300 (pre-implantation embryos and trophoblast outgrowths) or 1:400 (placental sections) for $1 \mathrm{~h}$ at RT. Tissues were counterstained with DAPI (Sigma-Aldrich, B2388) in PBS for $10 \mathrm{~min}$. Blastocysts were transitioned through increasing concentrations of glycerol prior to mounting with the antifading agent Fluoroshield (Sigma, F6182).

\section{Imaging and analysis}

Embryos and outgrowths were visualised using an inverted confocal microscope (Leica, DMi8). Pre-implantation embryos utilised a $40 \times$ air objective $(0.85 \mathrm{NA}$, pinhole airy $=1)$ and captured in $z$-stacks of 40 . Outgrowths were imaged at $20 \times$ in $z$-stacks of ten in a tile scan. Excitation filters of $488 \mathrm{~nm}$ (green), $405 \mathrm{~nm}$ (blue) and $561 \mathrm{~nm}$ (red) fluorescence were imaged separately. CDX2 immunoreactivity (green) was localised to the TE of the E5 blastocyst. Cells devoid of CDX2 staining were assumed to be ICM. TE and ICM cells were counted individually using ImageJ (NIH). ICM percentage was calculated as the number of ICM cells divided by total cells $\times 100$. TE nuclear CDX2 fluorescence was determined using the imaging processing tool Imaris (version 7.7.2, Bitplane). A mask was created around the DAPI channel using standard surface area detail and thresholds. CDX2-negative cells were deleted (being ICM) and adjoining nuclei were separated. CDX2 fluorescence intensity was measured within each nucleus, with all nuclear fluorescence summed to create the final data.

For trophoblast cell counts and ploidy analysis, $z$-stacks of the DAPI channel were compressed using 'average intensity' in ImageJ. ICM cells, as indicated by negative staining of pan-cytokeratin, were edited out using Photoshop. The smallest trophoblasts with positive staining for pancytokeratin had nuclear sizes exceeding $200 \mu \mathrm{m}^{2}$. ICM cells $(2 \mathrm{~N})$ were found to be less than $200 \mu \mathrm{m}^{2}$ in size. Nuclear sizes above $1000 \mu \mathrm{m}^{2}$ were considered P-TGCs. DNA content, a surrogate marker of ploidy, was quantified from fluorescence intensity of nuclear DAPI. DNA content of all trophoblasts and P-TGCs were normalised to ICM cells of the same image to obtain ploidy relative to $2 \mathrm{~N}$.

Placental cryosections were imaged on an upright fluorescence microscope (BX61 Olympus) using DAPI, GFP and Cy3 filters at $4 \times$ and $10 \times$ magnification using set parameters. Placentae were measured for average distance of trophoblast invasion from the P-TGC layer (identified by large polyploid nuclei), to the most distant pan-cytokeratin-positive SpATGC. Ploidy of uterine SpA-TGCs was assessed in Imaris as described above. No invasion was seen in all sections regardless of treatment after section 6 ( 1 in 10 sections were sampled at $10 \mu \mathrm{m}$, so section 6 correlates to $600 \mu \mathrm{m}$ into the block). 


\section{Post-mortem cohort studies}

For cohorts following E5, dams were returned to laboratory chow until postmortem analysis at E6 ( $n=3 /$ group), E7 ( $n=5 /$ group), E11 ( $n=4 /$ group), E13 ( $n=8 /$ group), E15 ( $n=15 /$ group) or E20 ( $n=6 /$ group). Maternal blood was taken from trunk after guillotine (E5-E11), or cardiac puncture following anaesthesia with 50:50 ketamine/xylazine as previously described (Gårdebjer et al., 2014). Blood was collected into EDTA- or heparin-coated tubes, and plasma was collected. For E6 and E7, implantation sites were dissected and taken whole for histological or molecular studies. Cohorts from E11 to E20 were dissected, and taken with the uterus attached for histology, or without for molecular studies. Fetuses and placentae were weighed at E13 and E15. At E15, additional measurements for placental dimensions were taken, and placentae were separated for decidua, junctional zone and labyrinth, and weighed separately. All analyses were carried out for both male and female fetuses with genotyping for sex performed as previously described (KalischSmith et al., 2017b).

\section{Histology and stereology for paraffin sections}

Stereology of uterine stromal volume and primary decidual volume was assessed on E7 implantation sites, whereas placental compartments; total, decidua, junctional zone, labyrinth, FBS and MBS were estimated at E13, E15 and E20, using the Cavalieri principle as described by Coan et al. (2004), and analysed in ImageJ (NIH). Uteri and placental halves were sectioned at $5 \mu \mathrm{m}$. For uteri, five to ten equally spaced sections (every 10th section) were selected from the midline of the embryo and either underwent in situ hybridisation (ISH) for Prl8a2 to detect the primary decidual zone of the uterus (Dai et al., 2002), or immunohistochemistry for vimentin to localise uterine stroma in the rat (Glasser and Julian, 1986; Korgun et al., 2007). Placental halves were sectioned at $5 \mu \mathrm{m}$ for five or six equally spaced sections with sampling fractions of 1:50 (E13), 1:100 (E15) and 1:150 (E20) used. Haematoxylin and Eosin (H\&E) staining was used for E13 placentae, Mest ISH for E15 placentae, and IsolectinB4 (1:200; conjugated, Sigma, L5391, derived from Bandeiraea simplicifolia) for E20 samples, the latter two of which both marked fetal endothelial cells of the FBS. Volume and surface areas of placental compartments were estimated. ISH for Prf 1 was also used to localise maternal dNK cells on midline sections. For all procedures, slides were dewaxed in xylene and rehydrated in an ethanol series.

\section{H\&E staining}

Slides were stained in Meyers Haematoxylin for 2 min prior to extensive washing in $\mathrm{dH}_{2} \mathrm{O}$. Slides were incubated in $1 \%$ alcoholic Eosin for $15 \mathrm{~min}$ prior to mounting in DPX mounting medium (Ajax Finechem).

\section{Immunohistochemistry and lectin histochemistry}

For immunohistochemistry, slides underwent antigen retrieval in sodium citrate buffer ( $10 \mathrm{mM}$ sodium citrate, $0.05 \%$ Tween $20, \mathrm{pH} 6.0$ ) for $2 \mathrm{~h}$ in a pressure cooker. Slides were washed three times in $1 \times$ PBS for $5 \mathrm{~min}$, blocked for endogenous peroxidases using $0.9 \% \mathrm{H}_{2} \mathrm{O}_{2}$ in $\mathrm{dH}_{2} \mathrm{O}$ for $10 \mathrm{~min}$, and washed (three times in $1 \times$ PBS for $5 \mathrm{~min}$ ). Slides were blocked in PBT for $1 \mathrm{~h}$ at RT. E7 samples were incubated with anti-rabbit vimentin (Thermo Fisher, PA5-27231; 1:200) at $4{ }^{\circ} \mathrm{C}$ overnight. The following day, slides were washed prior to incubation in biotinylated anti-rabbit secondary antibody (Vector Laboratories, PK-6101, 1:200) for $1 \mathrm{~h}$ at $37^{\circ} \mathrm{C}$. Slides were washed prior to incubation in Avidin-Biotin complex (Vector Laboratories, PK6100) for $1 \mathrm{~h}$ at $37^{\circ} \mathrm{C}$. Slides were washed and chromogen stain was developed using ImmPACT NovaRED Peroxidase Substrate (Vector Laboratories, SK-4805) as per manufacturer's protocol. For lectin histochemistry, slides underwent antigen retrieval and blocking steps as above. Slides were incubated in $0.1 \%$ Triton X-100 with $0.1 \mathrm{mM}$ ions $\left(\mathrm{MgCl}_{2}, \mathrm{CaCl}_{2}, \mathrm{MnCl}_{2}\right)$ for 10 min, prior to incubation of with IsolectinB4 (conjugated, Sigma, L5391, derived from Bandeiraea simplicifolia; 1:200) in $1 \times$ PBS for $2 \mathrm{~h}$ at RT. Slides were washed followed by chromogen development. Slides were counterstained with Haematoxylin and mounted.

\section{ISH}

ISH was carried out as previously described (Simmons et al., 2007, 2008). To construct ISH primers, firstly, gene sequences for the rat (Rattus norvegicus) were selected in the database Ensembl (Oxford). Primers were then designed with the program Primer3 (NIH) (see Table S5). cDNA probe templates were isolated by gel extraction (QIAquick Gel Extraction Kit, Qiagen). Digoxigenin labelling of cRNA probes was carried out as per manufacturer's instructions (Roche). Probe (1:200) hybridisation was carried out overnight at $65^{\circ} \mathrm{C}$ in a humidified chamber and included negative antisense (T3) controls.

\section{Electron microscopy}

Labyrinth midline samples from placentae (E15 and E20) were cut into $1 \mathrm{~mm}^{3}$ blocks and fixed until processing in $2.5 \%$ glutaraldehyde in $0.2 \mathrm{M}$ sodium cacodylate buffer. Samples were washed in $0.1 \mathrm{M}$ sodium cacodylate buffer at $250 \mathrm{~W}$ and $1 \%$ osmium tetroxide in cacodylate buffer at $80 \mathrm{~W}$ in a vacuum microwave (Biowave, Pelco). Samples were rinsed in water at $250 \mathrm{~W}$ and counterstained in $2 \%$ uranyl acetate $(\mathrm{aq})$ at $150 \mathrm{~W}$ under vacuum. Samples were dehydrated in an ethanol series $(30 \%, 50 \%, 70 \%$, $90 \%, 100 \%, 100 \%)$ at $250 \mathrm{~W}$ and infiltrated in an epon resin series $(1: 3,1: 1$, $3: 1,100 \%, 100 \%)$ under vacuum. Resin was polymerised for $48 \mathrm{~h}$ at $60^{\circ} \mathrm{C}$. Thick $(500 \mathrm{~nm})$ sections were cut and stained with Toluidine Blue to confirm localisation of the labyrinth. Thin sections of $100 \mathrm{~nm}$ were cut and placed onto copper grids, post-stained with lead citrate and visualised using an electron microscope (Jeol JEM-101). Two blocks per labyrinth samples were used to gather a minimum of six interhemal membranes to measure thickness, and averaged per block.

\section{Culture of mouse trophoblast stem cells}

Mouse trophoblast stem cells (RS26 line), were seeded at 10,000 cells per well and incubated in $0 \%$ (control), $0.2 \%$ or $1 \%$ ethanol as previously described (Kalisch-Smith et al., 2016). Cell were harvested at 2 days of differentiation prior to RNA extraction and QPCR, for these procedures (see Kalisch-Smith et al., 2016). Cells showed no signs of contamination.

\section{RNA extraction, CDNA synthesis and RT-PCR}

RNA was extracted from E15 placentae using RNeasy Mini Kit (Qiagen), as per manufacturer's protocol, with on-column DNase digestion. Extractions from other tissues used TRIzol (Thermo Fisher Scientific). GlycoBlue (1 $\mu \mathrm{l}$; Ambion) was added in the isopropanol step of trophoblast outgrowth extractions, and precipitated overnight at $-80^{\circ} \mathrm{C}$ due to low abundance of RNA and for clear localisation of the RNA pellet. Outgrowths also used the gDNA wipe-out step in the reverse transcription Quantitek kit (Qiagen), whereas E11 and E13 samples underwent DNase digestion with RNA cleanup on Qiagen columns. RNA purity was assessed using NanoDrop ND1000 spectrophotometer (NanoDrop Technologies), with A260:A280 nm absorbance ratio values between 1.8 and 2.1 indicating pure RNA samples. Quantities of RNA greater than $500 \mathrm{ng}$ were used for complementary DNA (cDNA) synthesis and gene analysis. RT-PCR was carried out using TaqMan and SYBR expression assays. For TaqMan, $1 \mu \mathrm{l}$ (100-200 ng) cDNA with $5 \mu 1$ TaqMan (Qiagen), $0.1 \mu 1$ TaqMan assay-on-demand primers (Thermo Fisher). The remaining volume was made up to $10 \mu \mathrm{l}$ with ultrapure water. SYBR reactions utilised $5 \mu \mathrm{l} \mathrm{SYBR}$ green reagent (Qiagen) with $3 \mu 1 \mathrm{H}_{2} \mathrm{O}$, $1 \mu \mathrm{l}$ of $4 \mu \mathrm{M}$ forward and reverse primers, and $1 \mu \mathrm{l}$ (100-200 ng) cDNA. Assays ran on a StepOne Real-Time PCR System (Applied Biosystems), for $1 \mathrm{~h}$. At least two housekeeping genes were used at each gestational age with a geometric mean taken (18S, Bac, Rplp0, Rpl13a, Rpl19). Results were used to calculate relative gene expression using the $\triangle \Delta \mathrm{CT}$ method (Moritz et al., 2002), standardised to control or control male group. SYBR primers were commercially available predesigned primer pairs from KiCqStart SYBR Green (Sigma). Melt curves were assessed to ensure amplification of a single product. Primer efficiencies were calculated from amplification data 'Delta Rn' and were plotted using the LinReg PCR program (version 11) (Ruijter et al., 2009). Primers, product codes and primer efficiencies are provided in Tables S5-S7.

\section{Western blot}

Total protein was extracted from uterine samples at E5 ( $n=5-6$ per treatment). Samples were homogenised in RIPA buffer and were blotted for the hormone receptors ESR1 $(60 \mathrm{kDa})$ and ESR2 $(55 \mathrm{kDa})$. Each sample of denatured protein $(40 \mu \mathrm{g})$ was loaded on a $12 \%$ polyacrylamide gel, and membranes were incubated overnight with anti-rabbit oestrogen receptor 
alpha (Abcam, ab32063; 1:1000) in 5\% non-fat milk/TBS-T (Tris-buffered saline, $0.1 \%$ Tween-20). The presence of both oestrogen receptor isoforms was detected. Anti-mouse $\beta$-actin (1:30,000, Sigma, A1978) was used as a control. Densitometric analysis was used to quantify bands using Image Studio Lite (LI-COR Biosciences).

\section{Radiotracer placental transport assays}

Dams were anaesthetised as described above and administered $50 \mu \mathrm{Ci} \mathrm{kg}^{-1}$ 3 - $O$-methyl-D- $\left[{ }^{3} \mathrm{H}\right]$-glucose (PerkinElmer, NET379001MC) in a $200 \mu \mathrm{l}$ bolus of PBS. Assays were carried out for 5 min prior to collection of maternal blood via cardiac puncture into EDTA-coated tubes, and dissection of placental and fetal tissues. Dams that showed unstable blood radioactivity from two consecutive samples were eliminated from analyses. Previous studies in the rat by Ericsson et al. (2007) showed no back-flux of these radiolabelled nutrients across the placenta for up to 7 min post-injection. Maternal plasma, fetal and placental tissues were digested overnight at $55^{\circ} \mathrm{C}$ in SOLVABLE (PerkinElmer). The following day, samples were decolourised with $0.2 \mathrm{ml} 30 \% \mathrm{H}_{2} \mathrm{O}_{2}$ for $30 \mathrm{~min}$. Scintillation fluid (Ultima Gold, PerkinElmer) was added and samples were counted for $\beta$-radioactivity in counts per minute (CPM) (Tri-Carb 3110 TR Beta Counter, PerkinElmer). Radioactivity for maternal plasma, placenta and fetal tissues were calculated for fetal accumulation (fetal CPM per g fetus), relative transport of placenta (fetal CPM per g placenta), placental uptake (placental CPM per g placenta) and placental clearance from maternal blood per unit time $\left(\mu 1 \mathrm{~min}^{-1} \mathrm{~g}^{-1}\right.$ placenta).

\section{Hormone analysis}

Progesterone concentrations were analysed in maternal plasma samples by radioimmunoassay (RIA) as previously described (Curlewis et al., 1985), except using progesterone antiserum C-9817 (Bioquest, North Ryde, NSW, Australia). Plasma samples were diluted (1:10) in charcoal-stripped fetal bovine serum (Gibco 12676, Invitrogen) and extracted in hexane prior to assay. The extraction efficiency was $75 \%$ and the values reported herein were not corrected for these losses. Assay sensitivity was $0.1 \mathrm{ng} / \mathrm{ml}$ and the intra- and inter-assay coefficients of variation were $8.1 \%$ and $7.9 \%$, respectively, for a quality control of $2.7 \mathrm{ng} / \mathrm{ml}$.

Plasma oestradiol-17 $\beta$ concentrations were measured using a commercially available RIA (DSL4800, Ultra-sensitive Oestradiol RIA, Beckman Coulter), following the manufacturer's instructions. Plasma samples were diluted (either 1:2 or 1:4) in charcoal-stripped fetal bovine serum. The assay sensitivity was $1.0 \mathrm{pg} / \mathrm{ml}$ and the intra- and inter-assay coefficients of variation were $0.6 \%$ and $6.2 \%$, respectively, for a quality control of $62.3 \mathrm{pg} / \mathrm{ml}$.

\section{Quantification of plasma choline levels}

E2 maternal plasma was collected at several intervals over $24 \mathrm{~h}$ following diet administration ( $30 \mathrm{~min}, 5 \mathrm{~h}$ and $21 \mathrm{~h}$ post-administration), and $\mathrm{E} 5$ plasma was collected at $09.00 \mathrm{~h}$ on the morning of E5 following sacrifice. All samples were stored at $-20^{\circ} \mathrm{C}$. Eighty microlitres of plasma was used to assess choline concentrations using the Choline Assay Kit (Abnova, KA1662) as per the manufacturer's instructions.

\section{DNA methylation analysis of placental samples}

To assess global DNA methylation, E13 whole placenta, as well as separated E15 and E20 labyrinth and junctional zones, were extracted for genomic DNA. DNA (100 ng) was assayed using the MethylFlash Methylated DNA Quantification Kit (Epigentek, P-1034) as per the manufacturer's instructions.

\section{Statistics}

For embryo studies analysing treatment (PC-EtOH, control) with sex, twoway ANOVA with Tukey's multiple comparison tests were used. For analyses comparing control and PC-EtOH treatments, Student's $t$-tests were used if data was normally distributed, or otherwise Mann-Whitney nonparametric tests were used. Data were tested for normality using a combination of tests (D'Agostino and Pearson, Shapiro-Wilk and Kolmogorov-Smirnov), prior to undergoing two-way ANOVA. Embryo parameters were pooled or taken as a litter average. For gene expression assays, mean relative expression of treatments were standardised to either the control group (uterine samples), or the control male group (if analysing treatment with sex). All data are displayed as mean \pm s.e.m. All graphs and statistics were performed using GraphPad Prism 6 software (GraphPad Software). $P \leq 0.05$ was considered significant.

\section{Acknowledgements}

Special thanks to Barb Arnts (University of Queensland Biological Resources) for assistance with tail vein injections, Jenn Kiel and Natalie Turner for tissue collection, and the PC-EtOH project students for animal treatment.

\section{Competing interests}

The authors declare no competing or financial interests.

\section{Author contributions}

Conceptualization: J.I.K.-S., D.G.S., M.P., M.E.W., K.M.M.; Methodology: J.I.K.-S., S.E.S., D.G.S., M.P., S.T.A., K.M.M.; Validation: J.I.K.-S.; Formal analysis: J.I.K.-S., S.E.S., S.T.A.; Investigation: J.I.K.-S., S.E.S., D.G.S., S.T.A., L.K.A., K.M.M.;

Resources: D.G.S., K.M.M.; Data curation: J.I.K.-S., K.M.M.; Writing - original draft: J.I.K.-S.; Writing - review \& editing: J.I.K.-S., D.G.S., M.P., S.T.A., L.K.A., M.E.W. K.M.M.; Supervision: D.G.S., M.P., K.M.M.; Project administration: K.M.M.; Funding acquisition: M.P., M.E.W., K.M.M

\section{Funding}

This project was funded by the National Health and Medical Research Counci (NHMRC) (APP1046137). J.I.K.-S. is a recipient of an Australian Postgraduate Award scholarship. K.M.M. is a Senior Research Fellow of the NHMRC.

\section{Supplementary information}

Supplementary information available online at

http://dev.biologists.org/lookup/doi/10.1242/dev.172205.supplemental

\section{References}

Ain, R., Dai, G., Dunmore, J. H., Godwin, A. R. and Soares, M. J. (2004). A prolactin family paralog regulates reproductive adaptations to a physiological stressor. Proc. Natl. Acad. Sci. USA 101, 16543-16548. doi:10.1073/pnas. 0406185101

Avalos, L. A., Roberts, S. C. M., Kaskutas, L. A., Block, G. and Li, D.-K. (2014). Volume and type of alcohol during early pregnancy and the risk of miscarriage. Subst. Use Misuse 49, 1437-1445. doi:10.3109/10826084.2014.912228

Barak, A. J., Beckenhauer, H. C. and Tuma, D. J. (1996). Betaine effects on hepatic methionine metabolism elicited by short-term ethanol feeding. Alcohol 13 483-486. doi:10.1016/0741-8329(96)00040-7

Bielawski, D. M., Zaher, F. M., Svinarich, D. M. and Abel, E. L. (2002). Paternal alcohol exposure affects sperm cytosine methyltransferase messenger RNA levels. Alcohol. Clin. Exp. Res. 26, 347-351. doi:10.1111/j.1530-0277.2002. tb02544.x

Blewitt, M. E., Gendrel, A.-V., Pang, Z., Sparrow, D. B., Whitelaw, N., Craig, J. M., Apedaile, A., Hilton, D. J., Dunwoodie, S. L., Brockdorff, N. et al. (2008). SmcHD1, containing a structural-maintenance-of-chromosomes hinge domain has a critical role in X inactivation. Nat. Genet. 40, 663-669. doi:10.1038/ng.142

Bloise, E., Lin, W., Liu, X., Simbulan, R., Kolahi, K. S., Petraglia, F., Maltepe, E., Donjacour, A. and Rinaudo, P. (2012). Impaired placental nutrient transport in mice generated by in vitro fertilization. Endocrinology 153, 3457-3467. doi:10. 1210/en.2011-1921

Bolnick, A. D., Bolnick, J. M., Kohan-Ghadr, H.-R., Kilburn, B. A., Hertz, M., Dai, J., Drewlo, S. and Armant, D. R. (2018). Nifedipine prevents apoptosis of alcohol-exposed first-trimester trophoblast cells. Alcohol. Clin. Exp. Res. 42 53-60. doi:10.1111/acer.13534

Burton, G. J. and Jauniaux, E. (2010). The maternal circulation and placental shape. In The Placenta and Human Developmental Programming (ed. G. J. Burton, D. J. P. Barker, A. Moffett and K. Thornburg), pp. 161-174. Cambridge: Cambridge University Press.

Burton, G. J. and Jauniaux, E. (2018). Pathophysiology of placental-derived fetal growth restriction. Am. J. Obstet. Gynecol. 218, S745-S761. doi:10.1016/j.ajog. 2017.11.577

Burton, G. J., Watson, A. L., Hempstock, J., Skepper, J. N. and Jauniaux, E. (2002). Uterine glands provide histiotrophic nutrition for the human fetus during the first trimester of pregnancy. J. Clin. Endocrinol. Metab. 87, 2954-2959. doi:10. 1210/jcem.87.6.8563

Burton, G. J., Fowden, A. L. and Thornburg, K. L. (2016). Placental origins of chronic disease. Physiol. Rev. 96, 1509-1565. doi:10.1152/physrev.00029.2015 Chakraborty, D., Rumi, M. A. K., Konno, T. and Soares, M. J. (2011). Natural killer cells direct hemochorial placentation by regulating hypoxia-inducible factor dependent trophoblast lineage decisions. Proc. Natl. Acad. Sci. USA 108 16295-16300. doi:10.1073/pnas.1109478108 
Chrysanthou, S., Senner, C. E., Woods, L., Fineberg, E., Okkenhaug, H., Burge, S., Perez-Garcia, V. and Hemberger, M. (2018). A critical role of TET1/2 proteins in cell-cycle progression of trophoblast stem cells. Stem Cell Rep. 10, 1355-1368. doi:10.1016/j.stemcr.2018.02.014

Coan, P. M., Ferguson-Smith, A. C. and Burton, G. J. (2004). Developmental dynamics of the definitive mouse placenta assessed by stereology. Biol. Reprod. 70, 1806-1813. doi:10.1095/biolreprod.103.024166

Coan, P. M., Ferguson-Smith, A. C. and Burton, G. J. (2005). Ultrastructura changes in the interhaemal membrane and junctional zone of the murine chorioallantoic placenta across gestation. J. Anat. 207, 783-796. doi:10.1111/j. 1469-7580.2005.00488.x

Coan, P. M., Vaughan, O. R., Sekita, Y., Finn, S. L., Burton, G. J., Constancia, M. and Fowden, A. L. (2010). Adaptations in placental phenotype support fetal growth during undernutrition of pregnant mice. J. Physiol. 588, 527-538. doi:10. 1113/jphysiol.2009.181214

Colvin, L., Payne, J., Parsons, D., Kurinczuk, J. J. and Bower, C. (2007). Alcohol consumption during pregnancy in nonindigenous west Australian women. Alcohol. Clin. Exp. Res. 31, 276-284. doi:10.1111/j.1530-0277.2006.00303.x

Corbel, C., Diabangouaya, P., Gendrel, A.-V., Chow, J. C. and Heard, E. (2013) Unusual chromatin status and organization of the inactive $\mathrm{X}$ chromosome in murine trophoblast giant cells. Development 140, 861-872. doi:10.1242/dev.087429

Curlewis, J. D., Axelson, M. and Stone, G. M. (1985). Identification of the majo steroids in ovarian and adrenal venous plasma of the brush-tail possum (Trichosurus vulpecula) and changes in the peripheral plasma levels of oestradiol and progesterone during the reproductive cycle. J. Endocrinol. 105, 53-62. doi:10.1677/joe.0.1050053

Dai, G., Lu, L., Tang, S., Peal, M. J. and Soares, M. J. (2002). Prolactin family miniarray: a tool for evaluating uteroplacental-trophoblast endocrine cell phenotypes. Reproduction 124, 755-765. doi:10.1530/rep.0.1240755

Ericsson, A., Säljö, K., Sjöstrand, E., Jansson, N., Prasad, P. D., Powell, T. L. and Jansson, T. (2007). Brief hyperglycaemia in the early pregnant rat increases fetal weight at term by stimulating placental growth and affecting placental nutrient transport. J. Physiol. 581, 1323-1332. doi:10.1113/jphysiol.2007.131185

Finer, L. B. and Zolna, M. R. (2016). Declines in unintended pregnancy in the United States, 2008-2011. N. Engl. J. Med. 374, 843-852. doi:10.1056/ NEJMsa1506575

Fisher, S. J. (2004). The placental problem: linking abnormal cytotrophoblas differentiation to the maternal symptoms of preeclampsia. Reprod. Biol. Endocrinol. 2, 53. doi:10.1186/1477-7827-2-53

Fleming, T. P., Kwong, W. Y., Porter, R., Ursell, E., Fesenko, I., Wilkins, A. Miller, D. J., Watkins, A. J. and Eckert, J. J. (2004). The embryo and its future. Biol. Reprod. 71, 1046-1054. doi:10.1095/biolreprod.104.030957

Gårdebjer, E. M., Cuffe, J. S. M., Pantaleon, M., Wlodek, M. E. and Moritz, K. M. (2014). Periconceptional alcohol consumption causes fetal growth restriction and increases glycogen accumulation in the late gestation rat placenta. Placenta $\mathbf{3 5}$, 50-57. doi:10.1016/j.placenta.2013.10.008

Gårdebjer, E. M., Anderson, S. T., Pantaleon, M., Wlodek, M. E. and Moritz, K. M. (2015). Maternal alcohol intake around the time of conception causes glucose intolerance and insulin insensitivity in rat offspring, which is exacerbated by a postnatal high-fat diet. FASEB J. 29, 2690-2701. doi:10.1096/fj.14-268979

Garro, A. J., Mcbeth, D. L., Lima, V. and Lieber, C. S. (1991). Ethanol consumption inhibits fetal DNA methylation in mice: implications for the fetal alcohol syndrome. Alcohol. Clin. Exp. Res. 15, 395-398. doi:10.1111/j.1530-0277.1991.tb00536.x

Glasser, S. R. and Julian, J. (1986). Intermediate filament protein as a marker of uterine stromal cell decidualization. Biol. Reprod. 35, 463-474. doi:10.1095/ biolreprod35.2.463

Hadjantonakis, A.-K., Cox, L. L., Tam, P. P. L. and Nagy, A. (2001). An X-linked GFP transgene reveals unexpected paternal X-chromosome activity in trophoblastic giant cells of the mouse placenta. Genesis 29, 133-140. doi:10 1002/gene.1016

Halsted, C. H., Villanueva, J. A., Devlin, A. M. and Chandler, C. J. (2002) Metabolic interactions of alcohol and folate. J. Nutr. 132, 2367S-2372S. doi:10. 1093/jn/132.8.2367S

Hamid, A., Wani, N. A., Rana, S., Vaiphei, K., Mahmood, A. and Kaur, J. (2007) Down-regulation of reduced folate carrier may result in folate malabsorption across intestinal brush border membrane during experimental alcoholism. FEBS J. 274, 6317-6328. doi:10.1111/j.1742-4658.2007.06150.x

Hannibal, R. L. and Baker, J. C. (2016). Selective amplification of the genome surrounding key placental genes in trophoblast giant cells. Curr. Biol. 26, 230-236. doi:10.1016/j.cub.2015.11.060

Haycock, P. C. and Ramsay, M. (2009). Exposure of mouse embryos to ethano during preimplantation development: effect on DNA methylation in the h19 imprinting control region. Biol. Reprod. 81, 618-627. doi:10.1095/biolreprod.108.074682

Higgins, J. S., Vaughan, O. R., Fernandez de Liger, E., Fowden, A. L. and Sferruzzi-Perri, A. N. (2016). Placental phenotype and resource allocation to fetal growth are modified by the timing and degree of hypoxia during mouse pregnancy. J. Physiol. 594, 1341-1356. doi:10.1113/JP271057

Hoki, Y., Ikeda, R., Mise, N., Sakata, Y., Ohhata, T., Sasaki, H., Abe, K. and Sado, T. (2011). Incomplete X-inactivation initiated by a hypomorphic Xist allele in the mouse. Development 138, 2649-2659. doi:10.1242/dev.061226
Hore, T. A., von Meyenn, F., Ravichandran, M., Bachman, M., Ficz, G., Oxley, D., Santos, F., Balasubramanian, S., Jurkowski, T. P. and Reik, W. (2016). Retinol and ascorbate drive erasure of epigenetic memory and enhance reprogramming to naïve pluripotency by complementary mechanisms. Proc. Natl. Acad. Sci. USA 113, 12202-12207. doi:10.1073/pnas.1608679113

Jones, K. L. and Smith, D. W. (1973). Recognition of the fetal alcohol syndrome in early infancy. Lancet 302, 999-1001. doi:10.1016/S0140-6736(73)91092-1

Kajantie, E., Thornburg, K. L., Eriksson, J. G., Osmond, C. and Barker, D. J. (2010). In preeclampsia, the placenta grows slowly along its minor axis. Int. J. Dev. Biol. 54, 469-473. doi:10.1387/ijdb.082833ek

Kalisch-Smith, J. I. and Moritz, K. M. (2017). Detrimental effects of alcohol exposure around conception: putative mechanisms. Biochem. Cell Biol. 96, 107-116.doi:10.1139/bcb-2017-0133

Kalisch-Smith, J. I., Outhwaite, J. E., Simmons, D. G., Pantaleon, M. and Moritz, K. M. (2016). Alcohol exposure impairs trophoblast survival and alters subtypespecific gene expression in vitro. Placenta 46, 87-91. doi:10.1016/j.placenta. 2016.08.080

Kalisch-Smith, J. I., Simmons, D. G., Dickinson, H. and Moritz, K. M. (2017a) Review: sexual dimorphism in the formation, function and adaptation of the placenta. Placenta 54, 10-16. doi:10.1016/j.placenta.2016.12.008

Kalisch-Smith, J. I., Simmons, D. G., Pantaleon, M. and Moritz, K. M. (2017b). Sex differences in rat placental development: from pre-implantation to late gestation. Biol. Sex Differ. 8, 17. doi:10.1186/s13293-017-0138-6

Kaminen-Ahola, N., Ahola, A., Maga, M., Mallitt, K.-A., Fahey, P., Cox, T. C. Whitelaw, E. and Chong, S. (2010). Maternal ethanol consumption alters the epigenotype and the phenotype of offspring in a mouse model. PLoS Genet. 6 , e1000811. doi:10.1371/journal.pgen.1000811

Kaminski, M., Rumeau, C. and Schwartz, D. (1978). Alcohol consumption in pregnant women and the outcome of pregnancy. Alcohol. Clin. Exp. Res. 2, 155-163. doi:10.1111/j.1530-0277.1978.tb04716.x

Kobor, M. S. and Weinberg, J. (2011). Focus on: epigenetics and fetal alcohol spectrum disorders. Alcohol Res. Health 34, 29-37.

Korgun, E. T., Cayli, S., Asar, M. and Demir, R. (2007). Distribution of laminin, vimentin and desmin in the rat uterus during initial stages of implantation. J. Mol. Histol. 38, 253-260. doi:10.1007/s10735-007-9095-4

Kwong, W. Y., Wild, A. E., Roberts, P., Willis, A. C. and Fleming, T. P. (2000) Maternal undernutrition during the preimplantation period of rat development causes blastocyst abnormalities and programming of postnatal hypertension. Development 127, 4195-4202.

Kwong, W. Y., Miller, D. J., Ursell, E., Wild, A. E., Wilkins, A. P., Osmond, C. Anthony, F. W. and Fleming, T. P. (2006). Imprinted gene expression in the rat embryo-fetal axis is altered in response to periconceptional maternal low protein diet. Reproduction 132, 265-277. doi:10.1530/rep.1.01038

Kwong, W. Y., Adamiak, S. J., Gwynn, A., Singh, R. and Sinclair, K. D. (2010) Endogenous folates and single-carbon metabolism in the ovarian follicle, oocyte and pre-implantation embryo. Reproduction 139, 705-715. doi:10.1530/REP-09 0517

Lawitts, J. A. and Biggers, J. D. (1993). Culture of preimplantation embryos Methods Enzymol. 225, 153-164. doi:10.1016/0076-6879(93)25012-Q

Li, Y., Seah, M. K. Y. and O'neill, C. (2016). Mapping global changes in nuclear cytosine base modifications in the early mouse embryo. Reproduction 151, 83-95. doi:10.1530/REP-15-0207

Lo, J. O., Schabel, M. C., Roberts, V. H. J., Wang, X., Lewandowski, K. S., Grant K. A., Frias, A. E. and Kroenke, C. D. (2017). First trimester alcohol exposure alters placental perfusion and fetal oxygen availability affecting fetal growth and development in a non-human primate model. Am. J. Obstet. Gynecol. 216 302.e1-302.e8. doi:10.1016/j.ajog.2017.01.016

Maconochie, N., Doyle, P., Prior, S. and Simmons, R. (2007). Risk factors for firs trimester miscarriage-results from a UK-population-based case-control study. BJOG 114, 170-186. doi:10.1111/j.1471-0528.2006.01193.x

Manning, M. A. and Eugene Hoyme, H. (2007). Fetal alcohol spectrum disorders: a practical clinical approach to diagnosis. Neurosci. Biobehav. Rev. 31, 230-238. doi:10.1016/j.neubiorev.2006.06.016

Marbury, M. C., Linn, S., Monson, R., Schoenbaum, S., Stubblefield, P. G. and Ryan, K. J. (1983). The association of alcohol consumption with outcome of pregnancy. Am. J. Public Health 73, 1165-1168. doi:10.2105/AJPH.73.10.1165

Mcgraw, S., Oakes, C. C., Martel, J., Cirio, M. C., DE Zeeuw, P., Mak, W., Plass, C., Bartolomei, M. S., Chaillet, J. R. and Trasler, J. M. (2013). Loss of DNMT10 disrupts imprinted $X$ chromosome inactivation and accentuates placental defects in females. PLoS Genet. 9, e1003873. doi:10.1371/journal.pgen.1003873

Meyer-Leu, Y., Lemola, S., Daeppen, J. B., Deriaz, O. and Gerber, S. (2011) Association of moderate alcohol use and binge drinking during pregnancy with neonatal health. Alcohol. Clin. Exp. Res. 35, 1669-1677. doi:10.1111/j.15300277.2011.01513.x

Milagre, I., Stubbs, T. M., King, M. R., Spindel, J., Santos, F., Krueger, F., Bachman, M., Segonds-Pichon, A., Balasubramanian, S., Andrews, S. R. et al. (2017). Gender differences in global but not targeted demethylation in iPSC reprogramming. Cell Rep. 18, 1079-1089. doi:10.1016/j.celrep.2017.01.008

Moritz, K. M., Johnson, K., Douglas-Denton, R., Wintour, E. M. and Dodic, M (2002). Maternal glucocorticoid treatment programs alterations in the renin- 
angiotensin system of the ovine fetal kidney. Endocrinology 143, 4455-4463. doi:10.1210/en.2002-220534

Mugford, J. W., Yee, D. and Magnuson, T. (2012). Failure of extra-embryonic progenitor maintenance in the absence of dosage compensation. Development 139, 2130-2138. doi:10.1242/dev.076497

Muggli, E., O'Leary, C., Donath, S., Orsini, F., Forster, D., Anderson, P. J., Lewis, S., Nagle, C., Craig, J. M., Elliott, E. et al. (2016). "Did you ever drink more?" A detailed description of pregnant women's drinking patterns. BMC Public Health 16, 683. doi:10.1186/s12889-016-3354-9

Müller, H., Liu, B., Croy, B. A., Head, J. R., Hunt, J. S., Dai, G. and Soares, M. J. (1999). Uterine natural killer cells are targets for a trophoblast cell-specific cytokine, prolactin-like protein A. Endocrinology 140, 2711-2720. doi:10.1210/ endo.140.6.6828

Naughton, C. A., Chandler, C. J., Duplantier, R. B. and Halsted, C. H. (1989). Folate absorption in alcoholic pigs: in vitro hydrolysis and transport at the intestinal brush border membrane. Am. J. Clin. Nutr. 50, 1436-1441. doi:10.1093/ajcn/50.6.1436

O'Leary, C. M., Nassar, N., Kurinczuk, J. J. and Bower, C. (2009). The effect of maternal alcohol consumption on fetal growth and preterm birth. BJOG 116 390-400. doi:10.1111/j.1471-0528.2008.02058.x

O'Neill, C. (1998). Endogenous folic acid is essential for normal development of preimplantation embryos. Hum. Reprod. 13, 1312-1316. doi:10.1093/humrep/13.5. 1312

Otero, N. K. H., Thomas, J. D., Saski, C. A., Xia, X. and Kelly, S. J. (2012). Choline supplementation and DNA methylation in the hippocampus and prefrontal cortex of rats exposed to alcohol during development. Alcohol. Clin. Exp. Res. 36, 1701-1709. doi:10.1111/j.1530-0277.2012.01784.x

Ouko, L. A., Shantikumar, K., Knezovich, J., Haycock, P., Schnugh, D. J. and Ramsay, M. (2009). Effect of alcohol consumption on CpG methylation in the differentially methylated regions of $\mathrm{H} 19$ and IG-DMR in male gametes: implications for fetal alcohol spectrum disorders. Alcohol. Clin. Exp. Res. 33 1615-1627. doi:10.1111/j.1530-0277.2009.00993.x

Ozanne, S. E. and Constância, M. (2007). Mechanisms of Disease: the developmental origins of disease and the role of the epigenotype. Nat. Clin Practice Endocrinol. Metab. 3, 539-546. doi:10.1038/ncpendmet0531

Padmanabhan, N., Jia, D., Geary-Joo, C., Wu, X., Ferguson-Smith, A. C., Fung, E., Bieda, M. C., Snyder, F. F., Gravel, R. A., Cross, J. C. et al. (2013). Mutation in folate metabolism causes epigenetic instability and transgenerational effects on development. Cell 155, 81-93. doi:10.1016/j.cell.2013.09.002

Pantaleon, M., Tan, H. Y., Kafer, G. R. and Kaye, P. L. (2010). Toxic effects of hyperglycemia are mediated by the hexosamine signaling pathway and o-linked glycosylation in early mouse embryos. Biol. Reprod. 82, 751-758. doi:10.1095/ biolreprod.109.076661

Refsum, H., Nurk, E., Smith, A. D., Ueland, P. M., Gjesdal, C. G., Bjelland, I., Tverdal, A., Tell, G. S., Nygård, O. and Vollset, S. E. (2006). The Hordaland Homocysteine Study: a community-based study of homocysteine, its determinants, and associations with disease. J. Nutr. 136, 1731S-1740S doi:10.1093/jn/136.6.1731S

Roberts, J. M. and Cooper, D. W. (2001). Pathogenesis and genetics of preeclampsia. Lancet 357, 53-56. doi:10.1016/S0140-6736(00)03577-7

Ruijter, J. M., Ramakers, C., Hoogaars, W. M. H., Karlen, Y., Bakker, O., van den Hoff, M. J. B. and Moorman, A. F. M. (2009). Amplification efficiency: linking baseline and bias in the analysis of quantitative PCR data. Nucleic Acids Res. 37 e45. doi:10.1093/nar/gkp045

Salihu, H. M., Kornosky, J. L., Lynch, O. N., Alio, A. P., August, E. M. and Marty P. J. (2011). Impact of prenatal alcohol consumption on placenta-associated syndromes. Alcohol 45, 73-79. doi:10.1016/j.alcohol.2010.05.010

Sandovici, I., Hoelle, K., Angiolini, E. and Constância, M. (2012). Placenta adaptations to the maternal-fetal environment: implications for fetal growth and developmental programming. Reprod. Biomed. Online 25, 68-89. doi:10.1016/j. rbmo.2012.03.017

Senner, C. E., Nesterova, T. B., Norton, S., Dewchand, H., Godwin, J., Mak, W. and Brockdorff, N. (2011). Disruption of a conserved region of Xist exon 1 impairs Xist RNA localisation and X-linked gene silencing during random and imprinted X chromosome inactivation. Development 138, 1541-1550. doi:10. 1242/dev.056812
Sferruzzi-Perri, A. N., Vaughan, O. R., Haro, M., Cooper, W. N., Musial, B. Charalambous, M., Pestana, D., Ayyar, S., Ferguson-Smith, A. C., Burton, G. J. et al. (2013). An obesogenic diet during mouse pregnancy modifies materna nutrient partitioning and the fetal growth trajectory. FASEB J. 27, 3928-3937. doi:10.1096/fj.13-234823

Simmons, D. G., Fortier, A. L. and Cross, J. C. (2007). Diverse subtypes and developmental origins of trophoblast giant cells in the mouse placenta. Dev. Biol. 304, 567-578. doi:10.1016/j.ydbio.2007.01.009

Simmons, D. G., Rawn, S. M., Davies, A., Hughes, M. and Cross, J. C. (2008) Spatial and temporal expression of the 23 murine Prolactin/Placental Lactogenrelated genes is not associated with their position in the locus. BMC Genomics 9 352. doi:10.1186/1471-2164-9-352

Sinclair, K. D., Allegrucci, C., Singh, R., Gardner, D. S., Sebastian, S., Bispham, J., Thurston, A., Huntley, J. F., Rees, W. D., Maloney et al. (2007). DNA methylation, insulin resistance, and blood pressure in offspring determined by maternal periconceptional B vitamin and methionine status. Proc. Natl. Acad. Sci. USA 104, 19351-19356. doi:10.1073/pnas.0707258104

Steegers-Theunissen, R. P. M., Twigt, J., Pestinger, V. and Sinclair, K. D. (2013). The periconceptional period, reproduction and long-term health of offspring: the importance of one-carbon metabolism. Hum. Reprod. Update 19, 640-655. doi:10.1093/humupd/dmt041

Strumpf, D., Mao, C. A., Yamanaka, Y., Ralston, A., Chawengsaksophak, K. Beck, F. and Rossant, J. (2005). Cdx2 is required for correct cell fate specification and differentiation of trophectoderm in the mouse blastocyst. Development 132, 2093-2102. doi:10.1242/dev.01801

Tai, M., Piskorski, A., Kao, J. C. W., Hess, L. A., de.La Monte, S. M. and Gundogan, F. (2016). Placental morphology in fetal alcohol spectrum disorders. Alcohol Alcohol. 52, 138-144. doi:10.1093/alcalc/agw088

Tan, K., An, L., Miao, K., Ren, L., Hou, Z., Tao, L., Zhang, Z., Wang, X., Xia, W., Liu, J. et al. (2016a). Impaired imprinted $X$ chromosome inactivation is responsible for the skewed sex ratio following in vitro fertilization. Proc. Natl. Acad. Sci. USA 113, 3197-3202. doi:10.1073/pnas.1523538113

Tan, K., Wang, Z., Zhang, Z., An, L. and Tian, J. (2016b). IVF affects embryonic development in a sex-biased manner in mice. Reproduction 151, 443-453. doi:10. 1530/REP-15-0588

Taylor, B. D., Ness, R. B., Klebanoff, M. A., Tang, G., Roberts, J. M., Hougaard, D. M., Skogstrand, K. and Haggerty, C. L. (2018). The impact of female fetal sex on preeclampsia and the maternal immune milieu. Pregnancy Hypertension 12 53-57. doi:10.1016/j.preghy.2018.02.009

Wallace, C., Burns, L., Gilmour, S. and Hutchinson, D. (2007). Substance use, psychological distress and violence among pregnant and breastfeeding Australian women. Aust. N Z J. Public Health 31, 51-56. doi:10.1111/j.1753 6405.2007.00010.x

Wallace, A. E., Fraser, R. and Cartwright, J. E. (2012). Extravillous trophoblast and decidual natural killer cells: a remodelling partnership. Hum. Reprod. Update 18 458-471. doi:10.1093/humupd/dms015

Wang, J. B., Mager, J., Chen, Y. J., Schneider, E., Cross, J. C., Nagy, A. and Magnuson, T. (2001). Imprinted $X$ inactivation maintained by a mouse Polycomb group gene. Nat. Genet. 28, 371-375. doi:10.1038/ng574

Watson, E. D. and Cross, J. C. (2005). Development of structures and transport functions in the mouse placenta. Physiology 20, 180-193. doi:10.1152/physiol. 00001.2005

Yan, J., Tanaka, S., Oda, M., Makino, T., Ohgane, J. and Shiota, K. (2001) Retinoic acid promotes differentiation of trophoblast stem cells to a giant cell fate. Dev. Biol. 235, 422-432. doi:10.1006/dbio.2001.0300

Zadora, J., Singh, M., Herse, F., Przybyl, L., Haase, N., Golic, M., Yung, H. W., Huppertz, B., Cartwright, J. E., Whitley, G. S. et al. (2017). Disturbed placenta imprinting in preeclampsia leads to altered expression of DLX5, a human-specific early trophoblast marker. Circulation 136, 1824-1839. doi:10.1161/ CIRCULATIONAHA.117.028110

Zeisel, S. H. (2006). Choline: critical role during fetal development and dietary requirements in adults. Annu. Rev. Nutr. 26, 229-250. doi:10.1146/annurev.nutr. 26.061505.111156

Zeisel, S. H. and da Costa, K.-A. (2009). Choline: an essential nutrient for public health. Nutr. Rev. 67, 615-623. doi:10.1111/j.1753-4887.2009.00246.x

Summary: Alcohol exposure in early pregnancy has sex-specific effects on placenta formation and function in the developing rat embryo.

Funding details

\begin{tabular}{llll}
\hline S.No. & Funder name & Funder ID & Grant ID \\
\hline 1 & National Health and Medical Research Council & http://dx.doi.org/10.13039/501100000925 & APP1046137 \\
\hline
\end{tabular}

